\title{
Fractional Laguerre Spectral Methods and Their Applications to Fractional Differential Equations on Unbounded Domain
}

\author{
T. Aboelenen ${ }^{* 1}$, Shaaban A. Bakr ${ }^{1,2}$ and H.M. El-Hawary ${ }^{1}$ \\ ${ }^{1}$ Department of Mathematics, Assiut University, Assiut 71516, Egypt \\ ${ }^{2}$ BCAM- Basque Center for Applied Mathematics, Mazarredo 14, E48009, Bilbao, Spain
}

\begin{abstract}
In this article, we first introduce a singular fractional Sturm-Liouville problem (SFSLP) on unbounded domain. The associated fractional differential operator is both Weyl and Caputo type. The properties of spectral data for fractional operator on unbounded domain have been investigated. Moreover, it has been shown that the eigenvalues of the singular problem are real-valued and the corresponding eigenfunctions are orthogonal. The analytical eigensolutions of SFSLP are obtained and defined as generalized Laguerre fractional-polynomials. The optimal approximation of such generalized Laguerre fractional-polynomials in suitably weighted Sobolev spaces involving fractional derivatives has been derived. We construct an efficient generalized Laguerre fractional-polynomials-Petrov-Galerkin (PG) methods for a class of fractional initial value problems (FIVPs) and fractional boundary value problems (FBVPs). As a numerical example, we examine space fractional advection-diffusion equation. Our theoretical results are confirmed by associated numerical results.
\end{abstract}

Keywords: singular fractional Sturm-Liouville operator, fractional-polynomials eigenfunctions, weighted Sobolev spaces, approximation results, Petrov-Galerkin spectral methods

2010 AMS Subject Classification: 65M70; 65N12; 34A08

\section{Introduction}

The differential operators of fractional order have gained a great development in both theory and application. It generalizes the concept of classical operators of integer orders to fractional orders. The notion of fractional derivative has been extended to many fractional ordinary differential equations (FODEs) such as fractional Cauchy equation, fractional Gauss equations [1]-[3] and fractional Sturm-Liouville equation [4], in addition to a variety of fractional partial differential equations (FPDEs) such as fractional Fokker-Planck equation [5], fractional Burgers equation [5], and fractional advection-diffusion equation [6]. In the last few decades, more and more scholars have realized FPDEs' important values in describing the real phenomenons and the field has acquired a huge development. A large amount of fractional models have been proposed and investigated in different aspects such as the advantages over the integer-order counterparts, analytical results, numerical techniques and so on.

The problems on bounded domains have been widely studied from various aspects. For the existence and analytical solutions to FPDEs in bounded domains [7]-[10] and references therein. From the numerical viewpoint, the FPDEs have been extensively concerned. Many powerful methods have been proposed, such as spectral method [11, 12], finite difference method [13]-[16], finite element method [17, random walk approach [18, the decomposition method [19, 20, the homotopy perturbation method 21, 222, the integral equation method 23]. and so many others.

Many scholars are today concerned with spectral methods for solving FPDEs. Lia and Xu [11] and [12] published

\footnotetext{
${ }^{*}$ Corresponding author. E-mail: tarek.aboelenen@aun.edu.eg .
} 
the first rigorous work on spectral methods for FPDEs in finite domains, who developed a time-space spectral method for the time-reactional diffusion equation. They accomplished exponential convergence in their numerical tests in agreement with their error analysis. Recently, exponentially accurate spectral and discontinuous hpspectral element methods of PG type for fractional differential equations (FDEs) have developed see [27]. These methods are developed based on the fractional Sturm-Liouville eigenproblems [28].

The collocation spectral method is applied to solve fractional differential equations, see [29]-31]. Meanwhile, the authors of [32, 33] applied the collocation spectral method for solving the fractional Burger and fractional KdV equation. Bhrawy and Abdelkawy 34 derived a general formulation for the Jacobi operational matrix of fractional derivatives, which is used to approximate numerical solution of a class of fractional differential equations. Recently, the tau spectral method is used together with the operational matrix of fractional derivatives to solve the linear fractional partial differential equation [35].

The research of fractional-order problems on unbounded domains is also of great interest. Many scholars are today concerned with the relevant analytical solutions for FDEs on unbounded domains. In [36]-38] investigated the existence of solutions of FODEs on unbounded domains based on the fixed point theorem combined with the diagonalization method . Langlands et al. 39 derived the solutions of fractional Nernst-Planck equations and derive fractional cable equations with different boundary conditions on infinite domains by using FourierLaplace transforms and their inverse transforms, where the solution was expressed using Fox $\mathrm{H}$ functions that seemed complex. Huang [40] considered the time-fractional telegraph equation. The fundamental solution for the Cauchy problem in a whole-space domain and Signaling problem in a half space domain is obtained by using the similar techniques. Gao et al. [16] considered fractional anomalous sub-diffusion equations on an unbounded domain. This paper main contribution lied in the reduction of fractional differential equations on an unbounded domain by using artificial boundary conditions and construction of the corresponding finite difference scheme with the help of method of order reduction. The generalized Laguerre spectral tau and collocation techniques were given in 41 to solve linear and nonlinear FDEs on the half line. These spectral techniques were developed and generalized by using the modified generalized Laguerre polynomials in [42]- 44 ].

The great difficulties to obtain the numerical solutions of differential problems on unbounded domains [45- 58 . Finite difference methods and finite element methods cannot be used directly. Numbers of scholars have agreed that spectral methods are one of the most popular ways to deal with the problems on unbounded domain, see [45]-[49]. For problems defined on the half-line and related problems, we often used the Laguerre spectral and pseudospectral methods; see [50]-[58, and the references therein. However, we noted that the eigensolutions of SFSLP on a semi-infinite interval have not been obtained explicitly and no numerical approximation results have been published so far in spite of importance of the numerical methods for FDEs on unbounded domains in practical applications. Until now, we are not aware of any published work on numerical solutions for FDEs on unbounded domains by using spectral methods. Thus, we dedicate this work to investigate the problem by using spectral methods based on the fractional Sturm-Liouville eigenproblem.

Our fundamental goal of this paper is to develop a spectral theory and numerical approximation for SFSLP on a semi-infinite interval. As a consequence, we introduce SFSLP on half line. We prove that the eigenvalues of this singular problem are real and the eigenfunctions corresponding to distinct eigenvalues are orthogonal; these too are computed analytically. We also show that these eigenfunctions are dense and forming a complete basis in the Hilbert space. In addition, these eigenfunctions are showing to be hierarchical. Next, we establish the analysis of approximations by generalized Laguerre fractional polynomials in weighted Sobolev spaces involving fractional derivatives. These results will be useful for error analysis of fractional spectral methods for FDEs on unbounded domains.

In the following, we first present some preliminary definitions of fractional calculus in Section 2. In Section 3, we proceed with the theory of SFSLP on half line. In Section 4, we establish the approximation results for generalized Laguerre fractional polynomials. In Sections 5, we construct efficient generalized Laguerre fractional polynomials PG methods for a class of FDEs, conduct error analysis and present ample supporting numerical results. We summarize our results in Section 6. 


\section{Preliminary definitions}

We start with some preliminary definitions of fractional calculus, see, e.g., [55].

\subsection{Liouville-Caputo Fractional Calculus}

The left-sided and right-sided Riemann-Liouville integrals of order $\mu$, when $0<\mu<1$, are defined, respectively, as

$$
\left({ }_{x_{L}}^{R L} \mathcal{I}_{x}^{\mu} f\right)(x)=\frac{1}{\Gamma(\mu)} \int_{x_{L}}^{x} \frac{f(s) d s}{(x-s)^{1-\mu}}, \quad x>x_{L},
$$

and

$$
\left({ }_{x}^{R L} \mathcal{I}_{x_{R}}^{\mu} f\right)(x)=\frac{1}{\Gamma(\mu)} \int_{x}^{x_{R}} \frac{f(s) d s}{(s-x)^{1-\mu}}, \quad x<x_{R},
$$

where $\Gamma$ represents the Euler gamma function. The corresponding inverse operators, i.e., the left-sided and right-sided fractional derivatives of order $\mu$, are then defined based on 2.1) and 2.2., as

$$
\left({ }_{x_{L}}^{R L} \mathcal{D}_{x}^{\mu} f\right)(x)=\frac{d}{d x}\left({ }_{x_{L}}^{R L} \mathcal{I}_{x}^{1-\mu} f\right)(x)=\frac{1}{\Gamma(1-\mu)} \frac{d}{d x} \int_{x_{L}}^{x} \frac{f(s) d s}{(x-s)^{\mu}}, \quad x>x_{L},
$$

and

$$
\left({ }_{x}^{R L} \mathcal{D}_{x_{R}}^{\mu} f\right)(x)=\frac{-d}{d x}\left({ }_{x}^{R L} \mathcal{I}_{x_{R}}^{1-\mu} f\right)(x)=\frac{1}{\Gamma(1-\mu)}\left(\frac{-d}{d x}\right) \int_{x}^{x_{R}} \frac{f(s) d s}{(s-x)^{\mu}}, \quad x<x_{R} .
$$

Furthermore, the corresponding left-sided and right-sided Caputo derivatives of order $\mu \in(0,1)$ are obtained as

$$
\left({ }_{x_{L}}^{C} \mathcal{D}_{x}^{\mu} f\right)(x)=\left({ }_{x_{L}}^{R L} \mathcal{I}_{x}^{1-\mu} \frac{d f}{d x}\right)(x)=\frac{1}{\Gamma(1-\mu)} \int_{x_{L}}^{x} \frac{f^{\prime}(s) d s}{(x-s)^{\mu}}, \quad x>x_{L},
$$

and

$$
\left({ }_{x}^{C} \mathcal{D}_{x_{R}}^{\mu} f\right)(x)=\left({ }_{x}^{R L} \mathcal{I}_{x_{R}}^{1-\mu} \frac{-d f}{d x}\right)(x)=\frac{1}{\Gamma(1-\mu)} \int_{x}^{x_{R}} \frac{-f^{\prime}(s) d s}{(x-s)^{\mu}}, \quad x<x_{R} .
$$

The two definitions of the left- and right-sided fractional derivatives of both Riemann-Liouville and Caputo type are linked by the following relationships, which can be derived by a direct calculation

$$
\begin{aligned}
& \left({ }_{x_{L}}^{R L} \mathcal{D}_{x}^{\mu} f\right)(x)=\frac{f\left(x_{L}\right)}{\Gamma(1-\mu)\left(x-x_{L}\right)^{\mu}}+\left({ }_{x_{L}}^{C} D_{x}^{\mu} f\right)(x), \\
& \left({ }_{x}^{R L} \mathcal{D}_{x_{R}}^{\mu} f\right)(x)=\frac{f\left(x_{R}\right)}{\Gamma(1-\mu)\left(x_{R}-x\right)^{\mu}}+\left({ }_{x}^{C} D_{x_{R}}^{\mu} f\right)(x) .
\end{aligned}
$$

\subsection{Weyl Fractional Calculus [56]}

The Weyl fractional integral of order $\mu, 0<\mu<1$ is defined as

$$
\left({ }_{x}^{W} \mathcal{I}_{\infty}^{\mu} f\right)(x)=\frac{1}{\Gamma(\mu)} \int_{x}^{\infty} \frac{f(s) d s}{(s-x)^{1-\mu}}
$$


The Weyl fractional derivative of order $0<\mu<1$ is defined as the left-inverse operator of the corresponding Weyl fractional integral

$$
\left({ }_{x}^{W} \mathcal{D}_{\infty}^{\mu} f\right)(x)=\frac{-d}{d x}\left({ }_{x}^{W} \mathcal{I}_{\infty}^{1-\mu} f\right)(x)
$$

Proposition 2.1 The fractional differential operator defined in (2.9)-(2.10) satisfy the following identity:

$$
\int_{0}^{\infty} g(x)_{x}^{W} \mathcal{D}_{\infty}^{\mu} f(x) d x=\int_{0}^{\infty} f(x)_{0}^{C} \mathcal{D}_{x}^{\mu} g(x) d x-\left[g(x){ }_{x}^{W} \mathcal{I}_{\infty}^{1-\mu} f(x)\right]_{x=0}^{\infty}
$$

\section{$3 \quad$ SFSLP on unbounded domain}

We formulate a fractional Sturm-Liouville problem on unbounded domain of order $2 \mu$ with fractional differential operator associated to Weyl and Caputo type of the same fractional-order $\mu$. This approach is motivated by the form of the integration by parts formulas (2.11).

Definition 3.1 Let $L^{\mu}$ be the singular fractional differential operator written as

$$
L^{\mu}:={ }^{W} \mathcal{D}^{\mu}\left[p(x)^{C} \mathcal{D}^{\mu}(.)\right] .
$$

Consider the singular fractional Sturm-Liouville equation

$$
L^{\mu} f(x)+\Lambda W(x) f(x)=0,
$$

where the fractional order $\mu \in(0,1)$ and ${ }^{W} \mathcal{D}^{\mu}={ }_{x}^{W} \mathcal{D}_{\infty}^{\mu}$ (i.e., Weyl fractional derivative of order $\mu$ ) and ${ }^{C} \mathcal{D}^{\mu}=$ ${ }_{0}^{C} \mathcal{D}_{x}^{\mu}$ (i.e., left-sided Caputo fractional derivative of order $\mu$ ), $p(x) \neq 0, W(x)>0 \forall x \in[0, \infty)$ and $p, W$ are real valued continuous functions in interval $[0, \infty)$. We shall solve 3.2 subject to a homogeneous Dirichlet and a homogeneous fractional integro-differential boundary conditions, defined as

$$
\begin{aligned}
& f(0)=0, \\
& \left\{{ }^{W} \mathcal{I}^{1-\mu}\left[p(x){ }^{C} \mathcal{D}^{\mu} f(x)\right]\right\}_{x=\infty}=0,
\end{aligned}
$$

where ${ }^{W} \mathcal{I}^{1-\mu}={ }_{x}^{W} \mathcal{I}_{\infty}^{1-\mu}$ (i.e., Weyl fractional integration of order $1-\mu$ ). The boundary conditions $(3.3)$ are natural in non-local calculus and FDEs on half line. In fact, the fundamental properties of eigensolutions and eigenvalues in the theory of classical Sturm-Liouville problems are related to the integration by parts formula for the first order derivatives and the choice of the boundary conditions. The problem of finding number $\Lambda$ such that the boundary-value problem $(\sqrt{3.2})-(\sqrt{3.3}))$ has a non-trivial solution. Such a value $\Lambda$ is called eigenvalue and the corresponding non-trivial solution is called eignsolution.

Theorem 3.1 Fractional differential operator (3.1) is self-adjoint on half line.

Proof. Let us consider the following equation

$$
\begin{aligned}
<L^{\mu} f, g>-<f, L^{\mu} g> & =\int_{0}^{\infty} L^{\mu} f(x) \cdot g(x) d x-\int_{0}^{\infty} f(x) \cdot L^{\mu} g(x) d x \\
& =\int_{0}^{\infty}{ }^{W} \mathcal{D}^{\mu}\left[p(x)^{C} \mathcal{D}^{\mu} f(x)\right] . g(x) d x-\int_{0}^{\infty}{ }^{W} \mathcal{D}^{\mu}\left[p(x)^{C} \mathcal{D}^{\mu} g(x)\right] . f(x) d x .
\end{aligned}
$$

By means of property 2.11) and boundary conditions (3.3), we obtain

$$
<L^{\mu} f, g>-<f, L^{\mu} g>=\int_{0}^{\infty} p(x){ }^{C} \mathcal{D}_{x}^{\mu} f(x) .{ }^{C} \mathcal{D}^{\mu} g(x) d x-\int_{0}^{\infty} p(x)^{C} \mathcal{D}^{\mu} g(x) .{ }^{C} \mathcal{D}^{\mu} f(x) d x=0 .
$$


Therefore

$$
<L^{\mu} f, g>=<f, L^{\mu} g>.
$$

Hence, $L^{\mu}$ is self-adjoint operator.

Theorem 3.2 The eigenvalues of SFSLP (3.2) are real valued on a semi-infinite interval.

Proof. Assume $\lambda$ is the eigenvalue of $(3.2)$ corresponding the eigenfunction $f(x)$

$$
L^{\mu} f(x)+\lambda W(x) f(x)=0,
$$

subject to the boundary conditions

$$
\begin{aligned}
& f(0)=0, \\
& \left\{{ }^{W} \mathcal{I}^{1-\mu}\left[p(x)^{C} \mathcal{D}^{\mu} f(x)\right]\right\}_{x=\infty}=0,
\end{aligned}
$$

and its complex conjugate $\hat{f}(x)$ and let $\hat{\lambda}$ is the eigenvalue of $\hat{f}(x)$

$$
L^{\mu} \hat{f}(x)+\hat{\lambda} W(x) \hat{f}(x)=0,
$$

corresponding to the following boundary conditions

$$
\begin{aligned}
& \hat{f}(0)=0, \\
& \left\{{ }^{W} \mathcal{I}^{1-\mu}\left[p(x)^{C} \mathcal{D}^{\mu} \hat{f}(x)\right]\right\}_{x=\infty}=0 .
\end{aligned}
$$

Now, we multiply (3.7) by $\hat{f}(x)$, and $(3.9)$ by $f(x)$ and subtract them, we get

$$
(\lambda-\hat{\lambda}) W(x) f(x) \hat{f}(x)=f(x) L^{\mu} \hat{f}(x)-\hat{f}(x) L^{\mu} f(x),
$$

integrating over the interval $[0, \infty)$ and utilizing the fractional integration-by-parts $(2.11)$, we obtain

$$
(\lambda-\hat{\lambda}) \int_{0}^{\infty} W(x)|f|^{2} d x=-\left\{f(x)^{W} \mathcal{I}^{1-\mu}\left[p(x)^{C} \mathcal{D}^{\mu} \hat{f}(x)\right]\right\}_{x=0}^{\infty}+\left\{\hat{f}(x)^{W} \mathcal{I}^{1-\mu}\left[p(x)^{C} \mathcal{D}^{\mu} f(x)\right]\right\}_{x=0}^{\infty}
$$

then, by applying the boundary conditions for $f(x)$ and $\hat{f}(x)$, we obtain

$$
(\lambda-\hat{\lambda}) \int_{0}^{\infty} W(x)|f|^{2} d x=0 .
$$

Therefore, $\lambda=\hat{\lambda}$ because $f$ is a non-trivial solution of the problem, and $W(x)$ is non-negative in interval $[0, \infty)$.

Theorem 3.3 The eigenfunctions corresponding to distinct eigenvalues of SFSLP (3.2) are orthogonal w.r.t. weight function $W(x)$ on $[0, \infty)$ that is

$$
\int_{0}^{\infty} W(x) f_{\lambda_{1}}(x) f_{\lambda_{2}}(x) d x=0, \quad \lambda_{1} \neq \lambda_{2},
$$

where function $f_{\lambda_{j}}$ corresponds to the eigenvalue $\lambda_{j}, j=1,2$.

Proof. Assume $f_{\lambda_{1}}(x)$ and $f_{\lambda_{2}}(x)$ are two eigenfunctions corresponding to two distinct eigenvalues $\lambda_{1}$ and $\lambda_{2}$, respectively:

$$
L^{\mu} f_{\lambda_{j}}(x)+\lambda_{j} W(x) f_{\lambda_{j}}(x)=0, \quad j=1,2 .
$$


Now, we obtain from Theorem 3.1

$$
<L^{\mu} f_{\lambda_{1}}, f_{\lambda_{2}}>=<f_{\lambda_{1}}, L^{\mu} f_{\lambda_{2}}>
$$

substitute (3.14) into 3.15), we obtain

$$
<-\lambda_{1} W(x) f_{\lambda_{1}}(x), f_{\lambda_{2}}(x)>=<f_{\lambda_{1}}(x),-\lambda_{2} W(x) f_{\lambda_{2}}(x)>,
$$

we can rewrite 3.16 as

$$
-\lambda_{1} \int_{0}^{\infty} W(x) f_{\lambda_{1}}(x) \cdot f_{\lambda_{2}}(x) d x=-\lambda_{2} \int_{0}^{\infty} W(x) f_{\lambda_{1}}(x) \cdot f_{\lambda_{2}}(x) d x,
$$

and since $\lambda_{1} \neq \lambda_{2}$, we obtain

$$
\int_{0}^{\infty} W(x) f_{\lambda_{1}}(x) f_{\lambda_{2}}(x) d x=0 .
$$

\subsection{Analytical eigensolutions to SFSLP on a semi-infinite interval}

Here, we obtain the analytical solution $f(x)$ to SFSLP 3.2 . Before that, we recall the following lemmas for the standard laguerre polynomials $L^{\alpha}(x)$.

Lemma 3.1 (See [57]) For $\mu>0, \alpha>-1$, and $x \in[0, \infty)$

$$
\frac{x^{\alpha+\mu} L_{n}^{\alpha+\mu}(x)}{\Gamma(n+\alpha+\mu+1)}=\frac{1}{\Gamma(\mu)} \int_{0}^{x} \frac{(x-y)^{\mu-1} y^{\alpha} L_{n}^{\alpha}(y)}{\Gamma(n+\alpha+1)} d y .
$$

By the left-sided Riemann-Liouville integral (2.1), we can rewrite (3.19) as

$$
{ }_{0}^{R L} \mathcal{I}_{x}^{\mu}\left[x^{\alpha} L_{n}^{\alpha}(x)\right]=\frac{\Gamma(n+\alpha+1)}{\Gamma(n+\alpha+\mu+1)} x^{\alpha+\mu} L_{n}^{\alpha+\mu}(x) .
$$

Lemma 3.2 (See [57]) For $\mu>0, \alpha>-1$, and $x \in[0, \infty)$

$$
e^{-y} L_{n}^{\alpha}(y)=\frac{1}{\Gamma(\mu)} \int_{y}^{\infty}(x-y)^{\mu-1} e^{-x} L_{n}^{\alpha+\mu}(x) d x .
$$

By the Weyl integral (2.9), we can re-write (3.21) as

$$
{ }_{x}^{W} \mathcal{I}_{\infty}^{\mu}\left[e^{-x} L_{n}^{\alpha+\mu}(x)\right]=e^{-x} L_{n}^{\alpha}(x) .
$$

Guo and Zhang [58] introduced a new family of generalized Laguerre polynomials, which is mutually orthogonal on the half-line, that we can use it to prove the following two lemmas:

Lemma 3.3 For $\mu>0, \alpha>-1, \beta \geq 1$, and $x \in[0, \infty)$

$$
\frac{x^{\alpha+\mu} L_{n}^{\alpha+\mu, \beta}(x)}{\Gamma(n+\alpha+\mu+1)}=\frac{1}{\Gamma(\mu)} \int_{0}^{x} \frac{(x-y)^{\mu-1} y^{\alpha} L_{n}^{\alpha, \beta}(y)}{\Gamma(n+\alpha+1)} d y .
$$

By the left-sided Riemann-Liouville integral (2.1), we can re-write 3.23 as

$$
{ }_{0}^{R L} \mathcal{I}_{x}^{\mu}\left[x^{\alpha} L_{n}^{\alpha, \beta}(x)\right]=\frac{\Gamma(n+\alpha+1)}{\Gamma(n+\alpha+\mu+1)} x^{\alpha+\mu} L_{n}^{\alpha+\mu, \beta}(x),
$$

we take the fractional derivative ${ }_{0}^{R L} \mathcal{D}_{x}^{\mu}$ on both sides of (3.24) to obtain

$$
{ }_{0}^{R L} \mathcal{D}_{x}^{\mu}\left[x^{\alpha+\mu} L_{n}^{\alpha+\mu, \beta}(x)\right]=\frac{\Gamma(n+\alpha+\mu+1)}{\Gamma(n+\alpha+1)} x^{\alpha} L_{n}^{\alpha, \beta}(x) .
$$


Lemma 3.4 For $\mu>0, \alpha>-1, \beta \geq 1$, and $x \in[0, \infty)$

$$
e^{-\beta y} L_{n}^{\alpha, \beta}(y)=\frac{\beta^{\mu}}{\Gamma(\mu)} \int_{y}^{\infty}(x-y)^{\mu-1} e^{-\beta x} L_{n}^{\alpha+\mu, \beta}(x) d x .
$$

By the Weyl integral (2.9), we can re-write (3.26) as

$$
{ }_{x}^{W} \mathcal{I}_{\infty}^{\mu}\left[e^{-\beta x} L_{n}^{\alpha+\mu, \beta}(x)\right]=\frac{e^{-\beta x} L_{n}^{\alpha, \beta}(x)}{\beta^{\mu}},
$$

we take the fractional derivative ${ }_{x}^{W} \mathcal{D}_{\infty}^{\mu}$ on both sides of (3.27) to obtain

$$
{ }_{x}^{W} \mathcal{D}_{\infty}^{\mu}\left[e^{-\beta x} L_{n}^{\alpha, \beta}(x)\right]=\beta^{\mu} e^{-\beta x} L_{n}^{\alpha+\mu, \beta}(x) .
$$

The singular fractional Sturm-Liouville equation for generalized laguerre fractional- polynomials with parameters $-1<\alpha<\mu-1$ and $\beta \geq 1$ is written as

$$
{ }^{W} \mathcal{D}^{\mu}\left[p(x)^{C} \mathcal{D}^{\mu} \mathcal{P}(x)\right]+\Lambda x^{\alpha-\mu+1} e^{-\beta x} \mathcal{P}(x)=0, x \in[0, \infty),
$$

subject to the boundary conditions

$$
\begin{aligned}
& \mathcal{P}(0)=0, \\
& \left\{{ }^{W} \mathcal{I}^{1-\mu}\left[p(x){ }^{C} \mathcal{D}^{\mu} \mathcal{P}(x)\right]\right\}_{x=\infty}=0,
\end{aligned}
$$

where $p(x)=x^{\alpha+1} e^{-\beta x}$. We also note that the weight function $W^{\alpha, \beta, \mu}(x)=x^{\alpha-\mu+1} e^{-\beta x}$ is a non-negative function.

Theorem 3.4 The eigenvalues of SFSLP (3.29) are real-valued, moreover, the eigenfunctions corresponding to distinct eigenvalues of SFSLP are orthogonal on a semi-infinite interval with respect to the weight function,

$$
W^{\alpha, \beta, \mu}(x)=x^{\alpha-\mu+1} e^{-\beta x}
$$

Proof. Part a: let $L^{\alpha, \beta ; \mu}$ be the fractional differential operator of order $2 \mu$ as

$$
L^{\alpha, \beta ; \mu}:={ }^{W} \mathcal{D}^{\mu}\left[p(x)^{C} \mathcal{D}^{\mu}(.)\right],
$$

and assume $\Lambda$ is the eigenvalue of 3.29 corresponding the eigenfunction $\psi(x)$.

$$
L^{\alpha, \beta ; \mu} \psi(x)+\Lambda W^{\alpha, \beta, \mu}(x) \psi(x)=0,
$$

subject to the boundary conditions

$$
\begin{aligned}
& \psi(0)=0, \\
& \left\{{ }^{W} \mathcal{I}^{1-\mu}\left[p(x)^{C} \mathcal{D}^{\mu} \psi(x)\right]\right\}_{x=\infty}=0,
\end{aligned}
$$

and its complex conjugate $\hat{\psi}(x)$ and $\hat{\Lambda}$ is the eigenvalue of $\hat{\psi}(x)$

$$
L^{\alpha, \beta ; \mu} \hat{\psi}(x)+\hat{\Lambda} W^{\alpha, \beta, \mu}(x) \hat{\psi}(x)=0,
$$

the corresponding boundary conditions are

$$
\begin{aligned}
& \hat{\psi}(0)=0, \\
& \left\{{ }^{W} \mathcal{I}^{1-\mu}\left[p(x){ }^{C} \mathcal{D}^{\mu} \hat{\psi}(x)\right]\right\}_{x=\infty}=0 .
\end{aligned}
$$

By multiply (3.32) by $\hat{\psi}(x)$, and 3.34 by $\psi(x)$ and subtract them, we get

$$
(\Lambda-\hat{\Lambda}) w^{\alpha, \beta, \mu}(x) \psi(x) \hat{\psi}(x)=\psi(x) L^{\alpha, \beta ; \mu} \hat{\psi}(x)-\hat{\psi}(x) L^{\alpha, \beta ; \mu} \psi(x),
$$


integrating over the interval $[0, \infty)$ and utilizing the fractional integration-by-parts (2.11), we obtain

$$
\begin{aligned}
&(\Lambda-\hat{\Lambda}) \int_{0}^{\infty} W^{\alpha, \beta, \mu}(x)|\psi|^{2} d x= \int_{0}^{\infty} p(x)^{C} \mathcal{D}^{\mu} \psi(x)^{C} \mathcal{D}^{\mu} \hat{\psi}(x) d x-\left\{\psi(x)^{W} \mathcal{I}^{1-\mu}\left[p(x)^{C} \mathcal{D}^{\mu} \hat{\psi}(x)\right]\right\}_{x=0}^{\infty} \\
&-\int_{0}^{\infty} p(x)^{C} \mathcal{D}^{\mu} \hat{\psi}(x)^{C} \mathcal{D}^{\mu} \psi(x) d x+\left\{\hat{\psi}(x)^{W} \mathcal{I}^{1-\mu}\left[p(x)^{C} \mathcal{D}^{\mu} \psi(x)\right]\right\}_{x=0}^{\infty}, \\
&(\Lambda-\hat{\Lambda}) \int_{0}^{\infty} W^{\alpha, \beta, \mu}(x)|\psi|^{2} d x=-\left\{\psi(x)^{W} \mathcal{I}^{1-\mu}\left[p(x)^{C} \mathcal{D}^{\mu} \hat{\psi}(x)\right]\right\}_{x=0}^{\infty}+\left\{\hat{\psi}(x)^{W} \mathcal{I}^{1-\mu}\left[p(x)^{C} \mathcal{D}^{\mu} \psi(x)\right]\right\}_{x=0}^{\infty},
\end{aligned}
$$

then, by applying the boundary conditions for $\psi(x)$ and $\hat{\psi}(x)$, we obtain

$$
(\Lambda-\hat{\Lambda}) \int_{0}^{\infty} W^{\alpha, \beta, \mu}(x)|\psi|^{2} d x=0 .
$$

Therefore, $\Lambda=\hat{\Lambda}$ because $\psi(x)$ is a non-trivial solution of the problem, and $W^{\alpha, \beta, \mu}(x)$ is non-negative in interval $[0, \infty)$.

Part b: Now, we prove the second statement on the orthogonality of the eigenfunctions with respect to the weight function $W^{\alpha, \beta, \mu}(x)$. Assume $\psi_{1}(x)$ and $\psi_{2}(x)$ are two eigenfunctions corresponding to two distinct eigenvalues $\Lambda_{1}$ and $\Lambda_{2}$, respectively. Then, they satisfy $(3.29)$ as

$$
L^{\alpha, \beta ; \mu} \psi_{i}(x)+\Lambda_{1} W^{\alpha, \beta, \mu}(x) \psi_{i}(x)=0, \quad i=1,2,
$$

subject to the boundary conditions

$$
\begin{aligned}
& \psi_{i}(0)=0, \\
& \left\{{ }^{W} \mathcal{I}^{1-\mu}\left[p(x)^{C} \mathcal{D}^{\mu} \psi_{i}(x)\right]\right\}_{x=\infty}=0, \quad i=1,2 .
\end{aligned}
$$

It can be shown that

$$
\left(\Lambda_{1}-\Lambda_{2}\right) W^{\alpha, \beta, \mu}(x) \psi_{1}(x) \psi_{2}(x)=\psi_{1}(x) L^{\alpha, \beta ; \mu} \psi_{2}(x)-\psi_{2}(x) L^{\alpha, \beta ; \mu} \psi_{1}(x),
$$

integrating over the interval $[0, \infty)$ and utilizing the fractional integration-by-parts (2.11), we obtain

$$
\left(\Lambda_{1}-\Lambda_{2}\right) \int_{0}^{\infty} W^{\alpha, \beta, \mu}(x) \psi_{1}(x) \psi_{2}(x) d x=-\left\{\psi_{1}(x)^{W} \mathcal{I}^{1-\mu}\left[p(x)^{C} \mathcal{D}^{\mu} \psi_{2}(x)\right]\right\}_{x=0}^{\infty}+\left\{\psi_{2}(x)^{W} \mathcal{I}^{1-\mu}\left[p(x)^{C} \mathcal{D}^{\mu} \psi_{1}(x)\right]\right\}_{x=0}^{\infty},
$$

then, by applying the boundary conditions for $\psi_{1}(x)$ and $\psi_{2}(x)$, and since $\Lambda_{1} \neq \Lambda_{2}$, we obtain

$$
\int_{0}^{\infty} W^{\alpha, \beta, \mu}(x) \psi_{1}(x) \psi_{2}(x) d x=0 .
$$

This ends the proof.

Theorem 3.5 The exact eigenfunctions of SFSLP (3.29) are given as

$$
\mathcal{P}(x)=\mathcal{P}_{n}^{(\alpha, \beta, \mu)}(x)=x^{-\alpha+\mu-1} L_{n-1}^{-\alpha+\mu-1, \beta}(x),
$$

and the corresponding distinct eigenvalues are

$$
\Lambda_{n}=-\frac{\Gamma(n-\alpha+\mu-1) \beta^{\mu}}{\Gamma(n-\alpha-1)} .
$$


Proof. First, from (3.45), it is clear that $\mathcal{P}_{n}^{(\alpha, \beta, \mu)}(0)=0$, we need to make sure that the other boundary condition is satisfied, by replacing the Caputo derivative by the Riemann-Liouville one, thanks to (2.7), we obtain

$$
\begin{aligned}
\left\{{ }^{W} \mathcal{I}^{1-\mu}\left[x^{\alpha+1} e^{-\beta x C} \mathcal{D}^{\mu} \mathcal{P}_{n}^{(\alpha, \beta, \mu)}(x)\right]\right\}_{x=\infty} & =\left\{{ }^{W} \mathcal{I}^{1-\mu}\left[x^{\alpha+1} e^{-\beta x R L} \mathcal{D}^{\mu} \mathcal{P}_{n}^{(\alpha, \beta, \mu)}(x)\right]\right\}_{x=\infty} \\
& =\left\{{ }^{W} \mathcal{I}^{1-\mu}\left[x^{\alpha+1} e^{-\beta x R L} \mathcal{D}^{\mu}\left(x^{-\alpha+\mu-1} L_{n-1}^{-\alpha+\mu-1, \beta}(x)\right)\right]\right\}_{x=\infty},
\end{aligned}
$$

(and by carrying out the fractional $R L$ derivative in the bracket using $(3.25)$ )

$$
\begin{aligned}
\left\{{ }^{W} \mathcal{I}^{1-\mu}\left[x^{\alpha+1} e^{-\beta x C} \mathcal{D}^{\mu} \psi_{n-1}^{\alpha, \beta, \mu}(x)\right]\right\}_{\infty} & =\left\{{ }^{W} \mathcal{I}^{1-\mu}\left[x^{\alpha+1} e^{-\beta x} \frac{\Gamma(n-\alpha+\mu)}{\Gamma(n+\alpha+1)} x^{-\alpha-1} L_{n-1}^{-\alpha-\beta}(x)\right]\right\}_{x=\infty} \\
& =\frac{\Gamma(n-\alpha+\mu)}{\Gamma(n+\alpha+1)}\left\{{ }^{W} \mathcal{I}^{1-\mu}\left[e^{-\beta x} L_{n-1}^{-\alpha-1, \beta}(x)\right]\right\}_{x=\infty},
\end{aligned}
$$

and by working out the fractional integration using Lemma 3.4 , we obtain

$$
\left\{{ }^{W} \mathcal{I}^{1-\mu}\left[x^{\alpha+1} e^{-\beta x C} \mathcal{D}^{\mu} \psi_{n-1}^{\alpha, \beta, \mu}(x)\right]\right\}_{\infty}=\frac{\Gamma(n-\alpha+\mu)}{\Gamma(n+\alpha+1) \beta^{1-\mu}}\left[e^{-\beta x} L_{n-1}^{-\alpha+\mu-2, \beta}(x)\right]_{x=\infty}=0 .
$$

The next step is to show that 3.45 satisfies 3.29 with eigenvalues 3.46 . So, we take a fractional integration of order $\mu$ on both sides of (3.29) and substitute (3.45). Then, again by replacing the Caputo derivative by the Riemann-Liouville one, thanks to 2.7), we obtain

$$
x^{\alpha+1} e^{-\beta x R L} \mathcal{D}^{\mu}\left[x^{-\alpha+\mu-1} L_{n-1}^{-\alpha+\mu-1, \beta}(x)\right]=-\Lambda_{n}{ }^{W} \mathcal{I}^{\mu}\left[e^{-\beta x} L_{n-1}^{-\alpha+\mu-1, \beta}(x)\right] .
$$

Finally, the fractional derivative on the left-hand side and the fractional integration on the right-hand side is worked out using (3.24) and (3.27) as

$$
\frac{\Gamma(n-\alpha+\mu-1)}{\Gamma(n-\alpha-1)} e^{-\beta x} L_{n-1}^{-\alpha-1, \beta}(x)=\frac{-\Lambda_{n}}{\beta^{\mu}} e^{-\beta x} L_{n-1}^{-\alpha-1, \beta}(x) .
$$

By a similar argument on the $e^{-x} L_{n-1}^{-\alpha-1, \beta}(x)$ being non-zero almost everywhere, we can cancel this term out on both sides and obtain

$$
\Lambda_{n}=\frac{-\Gamma(n-\alpha+\mu-1) \beta^{\mu}}{\Gamma(n-\alpha-1)}
$$

which shows that the eigenvalues of SFSLP are real-valued and discrete. In fact, this result agrees with Theorem 3.4. The orthogonality of the eigenfunctions with respect to $W^{\alpha, \beta, \mu}(x)=x^{\alpha-\mu+1} e^{-\beta x}$ is valid:

$$
\begin{aligned}
\int_{0}^{\infty} W^{\alpha, \beta, \mu}(x) \psi_{k}^{\alpha, \beta, \mu}(x) \psi_{j}^{\alpha, \beta, \mu}(x) d x & =\int_{0}^{\infty} W^{\alpha, \beta, \mu}(x)\left(x^{-\alpha+\mu-1}\right)^{2} L_{k-1}^{-\alpha+\mu-1, \beta}(x) L_{j-1}^{-\alpha+\mu-1, \beta}(x) d x \\
& =\int_{0}^{\infty} e^{-x} x^{-\alpha+\mu-1} L_{k-1}^{-\alpha+\mu-1, \beta}(x) L_{j-1}^{-\alpha+\mu-1, \beta}(x) d x \\
& =\kappa_{(k-1)}^{-\alpha+\mu-1, \beta} \delta_{k j},
\end{aligned}
$$

where

$$
\kappa_{(k-1)}^{-\alpha+\mu-1, \beta}=\frac{\Gamma(k-\alpha+\mu-1)}{\beta^{-\alpha+\mu}(k-1) !},
$$

where $\kappa_{k}^{-\alpha+\mu-1, \beta}$ is the orthogonality constant of the family of Laguerre polynomials with parameters $(-\alpha+\mu-$ $1, \beta)$, and this completes the proof.

The simplicity of the eigenvalues can be also shown in next theorem.

Theorem 3.6 Eigen-solutions of SFSLP (3.29) are dense in the Hilbert space and they forms a basis for $L_{W}^{2}[0, \infty)$. 
Proof. Let $P(x) \in L_{W}^{2}[0, \infty)$ then, $f(x)=x^{\alpha-\mu+1} P(x) \in L_{W}^{2}[0, \infty)$, as well when $\mu \in(0,1)$. Hence

$$
\begin{aligned}
\left\|\sum_{k=1}^{N} a_{k} \widetilde{\mathcal{P}}_{k}(x)-P(x)\right\|_{L_{W}^{2}[0, \infty)} & =\left\|\sum_{k=1}^{N} a_{k} x^{-\alpha+\mu-1} L_{k-1}^{-\alpha-\mu+1, \beta}(x)-P(x)\right\|_{L_{W}^{2}[0, \infty)} \\
& =\left\|x^{-\alpha+\mu-1} \sum_{k=1}^{N} a_{k} L_{k-1}^{-\alpha-\mu+1, \beta}(x)-x^{\alpha-\mu+1} P(x)\right\|_{L_{W}^{2}[0, \infty)} \\
& =\left\|x^{-\alpha+\mu-1} \sum_{k=1}^{N} a_{k} L_{k-1}^{-\alpha-\mu+1, \beta}(x)-f(x)\right\|_{L_{W}^{2}[0, \infty)} \| \\
& \leq\left\|x^{-\alpha+\mu-1}\right\|_{L_{W}^{2}[0, \infty)}\left\|\sum_{k=1}^{N} a_{k} L_{k-1}^{-\alpha-\mu+1, \beta}(x)-f(x)\right\|_{L_{W}^{2}[0, \infty)} \\
& \leq c\left\|\sum_{k=1}^{N} a_{k} L_{k-1}^{-\alpha-\mu+1, \beta}(x)-f(x)\right\|_{L_{W}^{2}[0, \infty)}, \\
\lim _{N \rightarrow \infty}\left\|\sum_{k=1}^{N} a_{k} \widetilde{\mathcal{P}}_{k}(x)-P(x)\right\|_{L_{W}^{2}[0, \infty)} & \leq \lim _{N \rightarrow \infty} c\left\|\sum_{k=1}^{N} a_{k} L_{k-1}^{-\alpha-\mu+1, \beta}(x)-f(x)\right\|_{L_{W}^{2}[0, \infty)}=0 .
\end{aligned}
$$

Therefore, $\sum_{k=1}^{N} a_{k} \widetilde{\mathcal{P}}_{k}(x) \rightarrow P(x)$ implying that $\left\{\widetilde{\mathcal{P}}_{k}(x): k=1,2, \ldots\right\}$ is dense and it forms a basis for $L_{W}^{2}[0, \infty)$. To show the simplicity of the eigenvalues, assume that corresponding to the eigenvalue $\Lambda_{k}$, there exits another eigenfunction $\widetilde{\mathcal{P}}_{k}^{*}(x) \in L_{W}^{2}[0, \infty)$ in addition to $\widetilde{\mathcal{P}}_{k}(x)$, which is by Theorem 3.4 orthogonal to the rest of the eigenfunctions $\widetilde{\mathcal{P}}_{n}(x), n \neq k$. By the density of the eigenfunctions set, i.e., 3.54), we can represent $\widetilde{\mathcal{P}}_{k}^{*}(x)$ as

$$
\widetilde{\mathcal{P}}_{k}^{*}(x)=\sum_{n=1}^{\infty} \hat{v}_{n} \widetilde{\mathcal{P}}_{n}(x) .
$$

By multiplying both sides by $\widetilde{\mathcal{P}}_{j}(x), j=1,2, \ldots$ and $j \neq k$, and integrating with respect to the weight function $W(x)$, we obtain

$$
\int_{0}^{\infty} W(x) \widetilde{\mathcal{P}}_{k}^{*}(x) \widetilde{\mathcal{P}}_{j}(x) d x=\sum_{n=1}^{\infty} \hat{v}_{n} \int_{0}^{\infty} W(x) \widetilde{\mathcal{P}}_{n}(x) \widetilde{\mathcal{P}}_{j}(x) d x=\hat{v}_{j} D_{j}
$$

this is contradicts to Theorem 3.4 Moreover, the eigenvalues $\Lambda_{n}$ are simple.

Theorem 3.7 Fractional differential operator in (3.31) is self-adjoint.

Proof. Let $y_{1}$ and $y_{2}$ be generalized Laguerre fractional-polynomials and consider the following equation:

$$
\begin{aligned}
<L^{\alpha, \beta ; \mu} y_{1}, y_{2}>-<y_{1}, L^{\alpha, \beta ; \mu} y_{2}> & =\int_{0}^{\infty} L^{\alpha, \beta ; \mu} y_{1} . y_{2} d x-\int_{0}^{\infty} y_{1} . L^{\alpha, \beta ; \mu} y_{2} d x \\
& =\int_{0}^{\infty}{ }^{W} \mathcal{D}^{\mu}\left[x^{\alpha+1} e^{-\beta x C} \mathcal{D}^{\mu} y_{1}\right] . y_{2} d x-\int_{0}^{\infty}{ }^{W} \mathcal{D}^{\mu}\left[x^{\alpha+1} e^{-\beta x C} \mathcal{D}^{\mu} y_{2}\right] . y_{1} d x
\end{aligned}
$$

By means of property (2.11), we obtain

$$
\begin{aligned}
<L^{\alpha, \beta ; \mu} y_{1}, y_{2}>-<y_{1}, L^{\alpha, \beta ; \mu} y_{2}>= & \int_{0}^{\infty} x^{\alpha+1} e^{-\beta x C} \mathcal{D}^{\mu} y_{1} \cdot{ }^{C} \mathcal{D}^{\mu} y_{2} d x-\left[y_{2}{ }^{W} \mathcal{I}^{1-\mu}\left(x^{\alpha+1} e^{-\beta x C} \mathcal{D}^{\mu} y_{1}\right)\right]_{x=0}^{\infty} \\
& -\int_{0}^{\infty} x^{\alpha+1} e^{-\beta x C} \mathcal{D}^{\mu} y_{2} \cdot{ }^{C} \mathcal{D}^{\mu} y_{1} d x+\left[y_{1}{ }^{W} \mathcal{I}^{1-\mu}\left(x^{\alpha+1} e^{-\beta x C} \mathcal{D}^{\mu} y_{2}\right)\right]_{x=0}^{\infty} .
\end{aligned}
$$


Now we consider $\psi_{n-1}^{\alpha, \beta, \mu}(x)$ generalized Laguerre fractional-polynomials, we also see that the weighting function $W^{\alpha, \beta, \mu}(x)=x^{\alpha-\mu+1} e^{-\beta x}$ and the property (2.7) helps in replacing ${ }^{C} \mathcal{D}^{\mu}$ by ${ }^{R L} \mathcal{D}^{\mu}$. Consequently

$$
\begin{aligned}
\left\{{ }^{W} \mathcal{I}^{1-\mu}\left[x^{\alpha+1} e^{-\beta x C} \mathcal{D}^{\mu} \psi_{n-1}^{\alpha, \beta, \mu}(x)\right]\right\}_{x=\infty} & =\left\{{ }^{W} \mathcal{I}^{1-\mu}\left[x^{\alpha+1} e^{-\beta x R L} \mathcal{D}^{\mu} \psi_{n-1}^{\alpha, \beta, \mu}(x)\right]\right\}_{x=\infty} \\
& =\left\{{ }^{W} \mathcal{I}^{1-\mu}\left[x^{\alpha+1} e^{-\beta x R L} \mathcal{D}^{\mu}\left(x^{-\alpha+\mu-1} L_{n-1}^{-\alpha+1, \beta}(x)\right)\right]\right\}_{x=\infty},
\end{aligned}
$$

(and by carrying out the fractional $R L$ derivative in the bracket using (3.25)

$$
\begin{aligned}
\left\{{ }^{W} \mathcal{I}^{1-\mu}\left[x^{\alpha+1} e^{-\beta x C} \mathcal{D}^{\mu} \psi_{n-1}^{\alpha, \beta, \mu}(x)\right]\right\}_{x=\infty} & =\left\{{ }^{W} \mathcal{I}^{1-\mu}\left[x^{\alpha+1} e^{-\beta x} \frac{\Gamma(n-\alpha+\mu)}{\Gamma(n+\alpha+1)} x^{-\alpha-1} L_{n-1}^{-\alpha-1, \beta}(x)\right]\right\}_{x=\infty} \\
& =\frac{\Gamma(n-\alpha+\mu)}{\Gamma(n+\alpha+1)}\left\{{ }^{W} \mathcal{I}^{1-\mu}\left[e^{-\beta x} L_{n-1}^{-\alpha-1, \beta}(x)\right]\right\}_{x=\infty},
\end{aligned}
$$

and by working out the fractional integration using Lemma 3.4, we obtain

$$
\left\{{ }^{W} \mathcal{I}^{1-\mu}\left[x^{\alpha+1} e^{-\beta x C} \mathcal{D}^{\mu} \psi_{n-1}^{\alpha, \beta}(x)\right]\right\}_{x=\infty}=\frac{\Gamma(n-\alpha+\mu)}{\Gamma(n+\alpha+1) \beta^{1-\mu}}\left[e^{-\beta x} L_{n-1}^{-\alpha+\mu-2, \beta}(x)\right]_{x=\infty}=0 .
$$

Hence

$$
\left\{y_{2}{ }^{W} \mathcal{I}^{1-\mu}\left[x^{\alpha+1} e^{-\beta x C} \mathcal{D}^{\mu} y_{1}(x)\right]\right\}_{x=0}^{\infty}=0,
$$

and

$$
\left\{y_{1}{ }^{W} \mathcal{I}^{1-\mu}\left[x^{\alpha+1} e^{-\beta x C} \mathcal{D}^{\mu} y_{2}(x)\right]\right\}_{x=0}^{\infty}=0 .
$$

Using $(3.62)$ and $(3.63)$, we obtain

$$
<L^{\alpha, \beta ; \mu} y_{1}, y_{2}>=<y_{1}, L^{\alpha, \beta ; \mu} y_{2}>.
$$

Hence, $L^{\alpha, \beta ; \mu}$ is self-adjoint operator.

\subsection{Properties of the eigen-solutions to SFSLP}

The basic properties of the generalized Laguerre fractional-polynomials are summarized below:

\section{- Fractional derivatives:}

$$
{ }^{R L} \mathcal{D}^{-\alpha+\mu+1} \mathcal{P}_{n}^{(\alpha, \beta, \mu)}(x)={ }^{C} \mathcal{D}^{-\alpha+\mu+1} \mathcal{P}_{n}^{(\alpha, \beta, \mu)}(x)=\frac{\Gamma(n-\alpha+\mu-1)}{\Gamma(n)} L_{n-1}^{0, \beta}(x) .
$$

\section{- First derivatives:}

$$
\frac{d}{d x} \mathcal{P}_{n}^{(\alpha, \beta, \mu)}(x)=(-\alpha+\mu-1) x^{-\alpha+\mu-2} L_{n-1}^{-\alpha+\mu-1, \beta}(x)-\beta x^{-\alpha+\mu-1} L_{n-2}^{-\alpha+\mu, \beta}(x) .
$$

We introduce the derivative operator:

$$
\hat{\partial}_{x}=\partial_{x}+(-\alpha+\mu-1) x^{-1} .
$$

It is clear that

$$
\partial_{x} L_{n-1}^{-\alpha+\mu-1, \beta}(x)=\hat{\partial}_{x} \mathcal{P}_{n}^{(\alpha, \beta, \mu)}(x),
$$

such that

$$
\partial_{x}^{k} L_{n}^{\alpha, \beta}(x)=(-1)^{k} L_{n-k}^{(\alpha+k, \beta)}(x), \quad n>k .
$$


- Recurrence relations: A recurrence relations is obtained for the generalized Laguerre fractional-polynomials

$$
\begin{aligned}
& \mathcal{P}_{1}^{(\alpha, \beta, \mu)}(x)=x^{-\alpha+\mu-1}, \\
& \mathcal{P}_{2}^{(\alpha, \beta, \mu)}(x)=x^{-\alpha+\mu-1}(-x-\alpha+\mu), \\
& \vdots \\
& \mathcal{P}_{n+1}^{(\alpha, \beta, \mu)}(x)=\left(a_{n}-b_{n} x\right) \mathcal{P}_{n}^{(\alpha, \beta, \mu)}(x)-c_{n} \mathcal{P}_{n-1}^{(\alpha, \beta, \mu)}(x), \\
& a_{n}=\frac{2 n-\alpha+\mu-2}{n}, \quad b_{n}=\frac{\beta}{n}, \quad c_{n}=\frac{n-\alpha+\mu-2}{n} . \\
& \mathcal{P}_{n}^{(\alpha, \beta, \mu)}(x)=\hat{\partial}_{x} \mathcal{P}_{n}^{(\alpha, \beta, \mu)}(x)-\hat{\partial}_{x} \mathcal{P}_{n+1}^{(\alpha, \beta, \mu)}(x), \\
& x \hat{\partial}_{x} \mathcal{P}_{n}^{(\alpha, \beta, \mu)}(x)=\frac{n-1}{\beta} \mathcal{P}_{n}^{(\alpha, \beta, \mu)}(x)-\frac{(n+\alpha-1)}{\beta} \mathcal{P}_{n-1}^{(\alpha, \beta, \mu)}(x) .
\end{aligned}
$$

- Orthogonality:

$$
\int_{0}^{\infty} W^{(\alpha, \beta, \mu)}(x) \mathcal{P}_{k}^{(\alpha, \beta, \mu)}(x) \mathcal{P}_{j}^{(\alpha, \beta, \mu)}(x) d x=\Theta_{k}^{\alpha, \beta, \mu} \delta_{k j}
$$

where

$$
\Theta_{k}^{\alpha, \beta, \mu}=\frac{\Gamma(k-\alpha+\mu)}{\beta^{-\alpha+\mu}(k-1) !} .
$$

\section{- Special values:}

$$
\mathcal{P}_{n}^{(\alpha, \beta, \mu)}(0)=1 .
$$

Lemma 3.5 The eigensolutions to SFSLP denoted by $\mathcal{P}_{n}^{(\alpha, \beta, \mu)}(x)$ are given by

$$
\mathcal{P}_{n}^{(\alpha, \beta, \mu)}(x)=\sum_{j=0}^{n-1} \frac{(-\beta)^{j}}{j !}\left(\begin{array}{c}
n-\alpha+\mu-2 \\
n-j-1
\end{array}\right) x^{j-\alpha+\mu-1}
$$

Definition 3.2 We define the finite-dimensional fractional-polynomial space:

$$
\mathbf{F}_{N}^{(\tilde{\mu}, \beta)}\left(\mathbb{R}_{+}\right)=\left\{\varphi=x^{\tilde{\mu}} \psi, \psi \in \mathbb{P}_{N}, \tilde{\mu} \in(0,1)\right\} .
$$

Taking $\tilde{\mu}=-\alpha+\mu-1$, then fractional-polynomial space $\mathbf{F}_{N}^{(\tilde{\mu}, \beta)}$ can be re-written as

$$
\mathbf{F}_{N}^{(\tilde{\mu}, \beta)}\left(\mathbb{R}_{+}\right)=F_{N}^{(\alpha, \beta, \mu)}\left(\mathbb{R}_{+}\right)=\left\{\varphi=x^{\tilde{\mu}} \psi, \psi \in \mathbb{P}_{N}\right\}=\operatorname{span}\left\{\mathcal{P}_{n}^{(\alpha, \beta, \mu)}: 0 \leq n \leq N\right\},
$$

such that $\mathbb{P}_{N}$ be the set of all algebraic (real-valued) polynomials of degree at most $N$.

Theorem 3.8 The eigensolutions to (3.29), $\mathcal{P}_{n}^{(\alpha, \beta, \mu)}(x), n \in N$ and $n<\infty$, form a complete hierarchical basis for the finite dimensional space of fractional-polynomials $F_{N}^{(\alpha, \beta, \mu)}$, where $\mu \in(0,1)$.

Proof. From Definition 3.2, we can re-write (3.74) as

$$
M \underline{x}=\underline{\mathcal{P}}^{(\alpha, \beta, \mu)},
$$


where

$$
\underline{x}=\left(\begin{array}{c}
x^{\mu-\alpha-1} \\
x^{\mu-\alpha} \\
\vdots \\
x^{n-1+\mu-\alpha-1}
\end{array}\right) \quad \text { and } \quad \underline{\mathcal{P}}^{(\alpha, \beta, \mu)}=\left(\begin{array}{c}
\mathcal{P}_{1}^{(\alpha, \beta, \mu)}(x) \\
\mathcal{P}_{2}^{(\alpha, \beta, \mu)}(x) \\
\vdots \\
\mathcal{P}_{n}^{(\alpha, \beta, \mu)}(x)
\end{array}\right) \text {, }
$$

and finally, $M=\left\{M_{j k}\right\}_{k, j=1}^{n}$ is an $n * n$ matrix obtained as

$$
M=\left\{M_{j k}\right\}_{k, j=1}^{n}=\left(\begin{array}{c}
n-\alpha+\mu-2 \\
n-j-1
\end{array}\right)
$$

which is a lower-triangular matrix. Thanks to the orthogonality of the $\mathcal{P}_{n}^{(\alpha, \beta, \mu)}(x)$, the eigenfunctions are linearly independent, therefore, the matrix $M$ is invertible. Let $\mathcal{M}=M^{-1}$, which is also lower triangular. Hence,

$$
\underline{x}=\mathcal{M} \underline{\mathcal{P}}^{(\alpha, \beta, \mu)} .
$$

Moreover, each element in the fractional-polynomials space $F_{N-1}^{(\alpha, \beta, \mu)}$, say $x^{m+\mu-\alpha-1}, 0 \leq m \leq n-1$, can be uniquely represented through the following expansion

$$
x^{m+\mu-\alpha-1}=\sum_{k=1}^{n} c_{k} \mathcal{P}_{k}^{(\alpha, \beta, \mu)}(x)=\sum_{k=1}^{n}\left\{\mathcal{M}_{m k}\right\} \mathcal{P}_{k}^{(\alpha, \beta, \mu)}(x)=\sum_{k=1}^{m}\left\{\mathcal{M}_{m k}\right\} \mathcal{P}_{k}^{(\alpha, \beta, \mu)}(x),
$$

where the last equality holds since $\mathcal{M}$ is a lower-triangular matrix. As seen in (3.76), the fractal expansion set $\mathbf{F}_{N}^{\mu-\alpha-1, \beta} \subset F_{N+1}^{(\alpha, \beta, \mu)}$, which indicates that the eigen-solutions $\mathcal{P}_{n}^{(\alpha, \beta, \mu)}(x)$ form a hierarchical expansion basis set.

From Fig. 1. we notice that the growth of magnitude in the eigenvalues of SFSLP, the optimal highest magnitude is achieved when $\alpha \longrightarrow-1, \forall \mu \in(0,1)$. The growth of the $\left|\Lambda_{n}\right|$ corresponding to two values of $\mu=0.5$, and $\mu=0.99$ is shown in Fig. 1 1 . The case $\mu=0.99$ leads to an exactly linear growth mode. We emphasize that $\mu=0.99$ grows linearly as opposed to quadratical growth in the Jacobi poly-fractonomials case [28. In Fig. 2 , we plot the eigenfunctions of SFSLP, $\mathcal{P}_{n}^{(\alpha, \beta, \mu)}(x)$, of different orders and corresponding to different values of $\mu$ used in Fig. 1. In a similar fashion, we compare the eigensolutions with the corresponding standard generalized Laguerre $L_{n}^{-\alpha+\mu-1, \beta}(x)$ in each plot.

\section{Approximation by generalized Laguerre fractional-polynomials}

The main concern of this section is to study the approximation properties of the family of generalized Laguerre fractional-polynomials and show that approximation by generalized Laguerre fractional-polynomials leads to typical spectral convergence for functions in appropriate weighted Sobolev spaces involving fractional derivatives. Such approximation results play a crucial role in the analysis of fractional spectral methods for FDEs.

We can study the approximation properties of the family of generalized Laguerre fractional-polynomials. In such setting, we can expand any $v \in L_{W^{(\alpha, \beta, \mu)}}^{2}\left(\mathbb{R}_{+}\right)$as

$$
v(x)=\sum_{n=1}^{\infty} \hat{v}_{n}^{(\alpha, \beta, \mu)} \mathcal{P}_{n}^{(\alpha, \beta, \mu)}(x),
$$

where

$$
\hat{v}_{n}^{(\alpha, \beta, \mu)}=\frac{1}{\Theta_{n}^{\alpha, \beta, \mu}} \int_{0}^{\infty} v(x) \mathcal{P}_{n}^{(\alpha, \beta, \mu)}(x) W^{(\alpha, \beta, \mu)}(x) d x .
$$



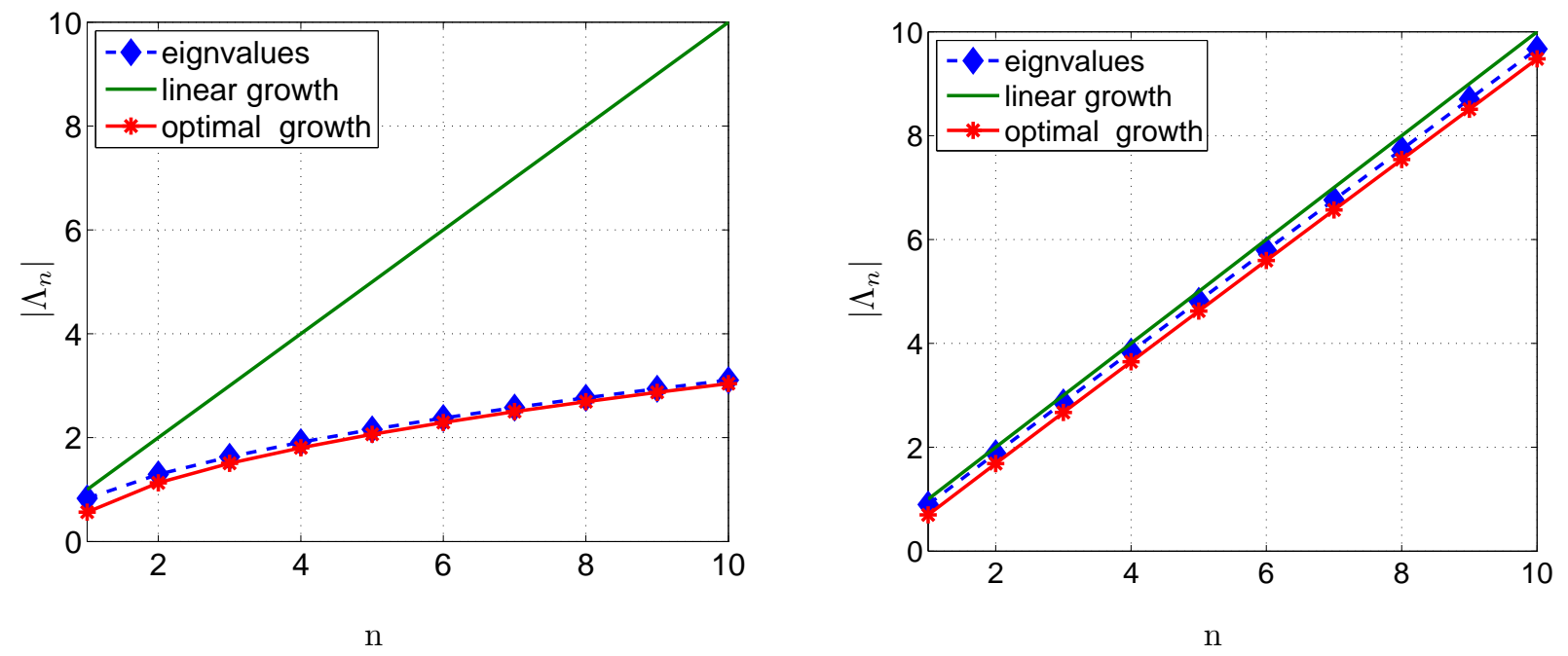

Figure 1: Magnitude of the eigenvalues of SFSLP, versus $n$, with $\alpha=-0.9$ and $\beta=1$. Left: $\mu=0.5$ (sublinear growth). Right: $\mu=0.99$ ( linear growth).

We now wish to estimate the rate of convergence of the eigenfunction expansion 4.13). By multiplying 4.13) by $L^{\alpha, \beta ; \mu} \mathcal{P}_{k}^{(\alpha, \beta, \mu)}(x), k=1,2, \ldots, N$, and integrating in the interval $[0, \infty)$, we obtain

$$
\int_{0}^{\infty} v(x) L^{\alpha, \beta ; \mu} \mathcal{P}_{k}^{(\alpha, \beta, \mu)}(x) d x=\int_{0}^{\infty}\left(\sum_{n=1}^{N} \hat{v}_{n} \mathcal{P}_{n}^{(\alpha, \beta, \mu)}(x)\right) L^{\alpha, \beta ; \mu} \mathcal{P}_{k}^{(\alpha, \beta, \mu)}(x) d x .
$$

Hence

$$
\int_{0}^{\infty} v(x) L^{\alpha, \beta ; \mu} \mathcal{P}_{k}^{(\alpha, \beta, \mu)}(x) d x=-\sum_{n=1}^{N} \hat{v}_{n} \Lambda_{n} \int_{0}^{\infty} x^{\alpha-\mu+1} e^{-\beta x} \mathcal{P}_{n}^{(\alpha, \beta, \mu)}(x) \mathcal{P}_{k}^{(\alpha, \beta, \mu)}(x) d x,
$$

and thanks to the orthogonality property $(3.71)$ we get

$$
\hat{v}_{k}=\frac{-1}{\Lambda_{k} \Theta_{k}^{\alpha, \beta, \mu}} \int_{0}^{\infty} v(x) L^{\alpha, \beta ; \mu} \mathcal{P}_{k}^{(\alpha, \beta, \mu)}(x) d x
$$

or equivalently by (3.31),

$$
\hat{v}_{k}=\frac{-1}{\Lambda_{k} \Theta_{k}^{\alpha, \beta, \mu}} \int_{0}^{\infty} v(x)^{W} \mathcal{D}^{\mu}\left[x^{\alpha+1} e^{-\beta x C} \mathcal{D}^{\mu} \mathcal{P}_{k}^{(\alpha, \beta, \mu)}(x)\right] d x .
$$

Now, by carrying out the fractional integration-by-parts 2.11, we get

$$
\hat{v}_{k}=\frac{-1}{\Lambda_{k} \Theta_{k}^{\alpha, \beta, \mu}} \int_{0}^{\infty} x^{\alpha+1} e^{-\beta x C} \mathcal{D}^{\mu} v(x)^{C} \mathcal{D}^{\mu} \mathcal{P}_{k}^{(\alpha, \beta, \mu)}(x) d x .
$$

Again, by the fractional integration-by-parts 2.11, we obtain

$$
\hat{v}_{k}=\frac{-1}{\Lambda_{k} \Theta_{k}^{\alpha, \beta, \mu}} \int_{0}^{\infty} \mathcal{P}_{k}^{(\alpha, \beta, \mu)}(x)^{W} \mathcal{D}^{\mu}\left[x^{\alpha+1} e^{-\beta x C} \mathcal{D}^{\mu} v(x)\right] d x
$$




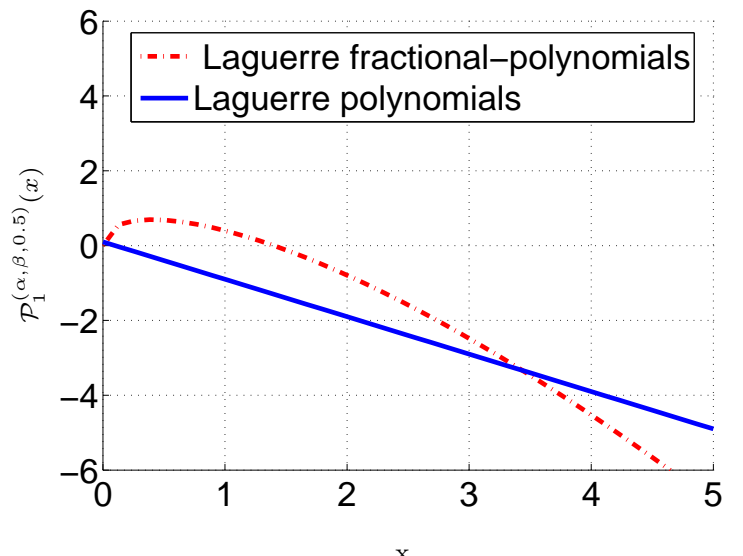

$\Lambda_{1}=0.7073, \mu=0.5$

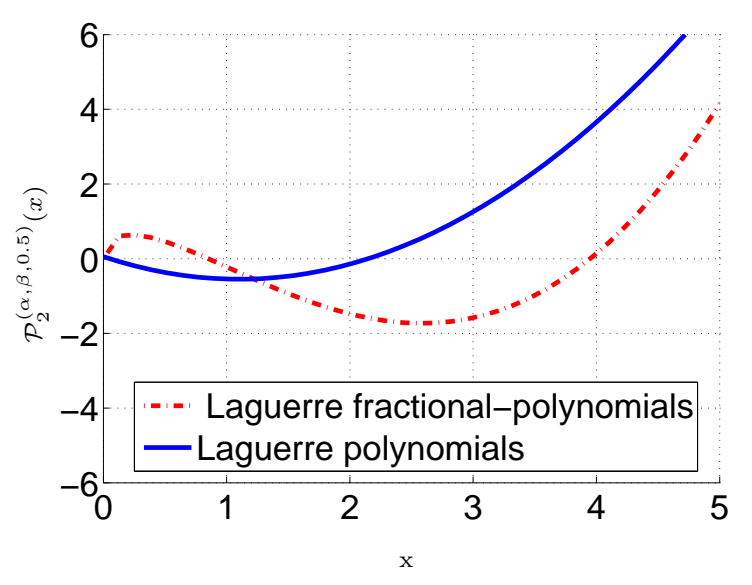

$\Lambda_{2}=1.2126, \mu=0.5$

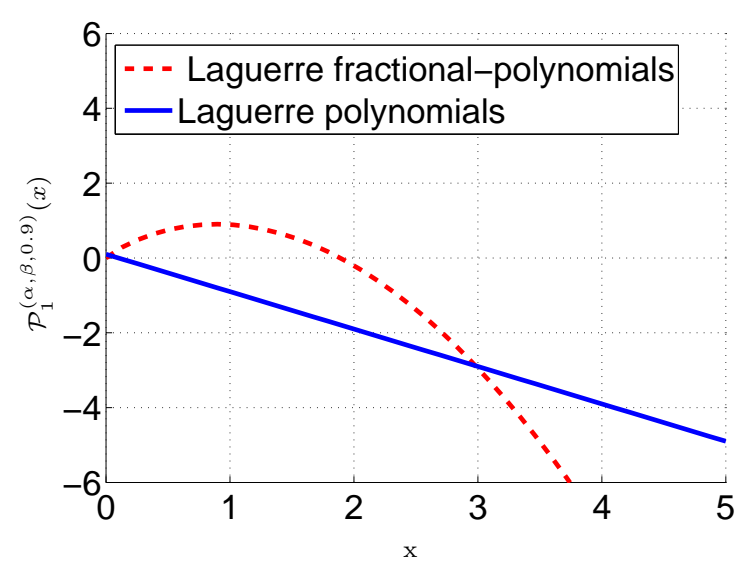

$\Lambda_{1}=0.8968, \mu=0.99$

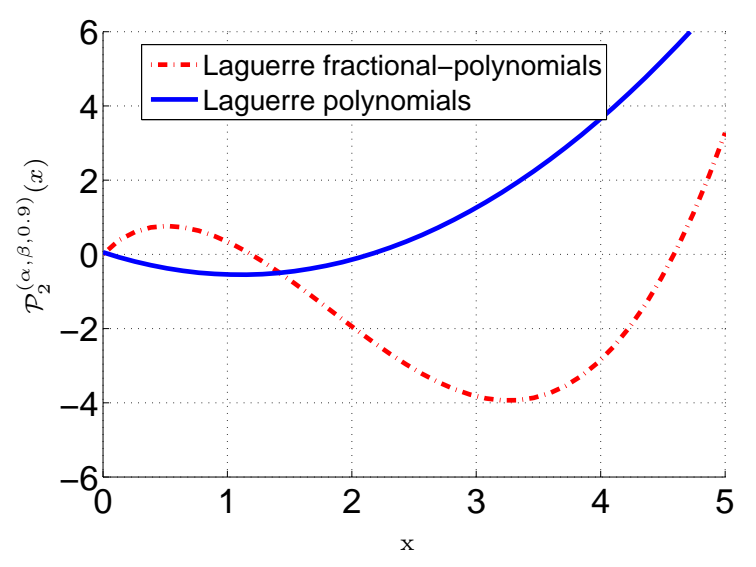

$\Lambda_{2}=1.8833, \mu=0.99$ 


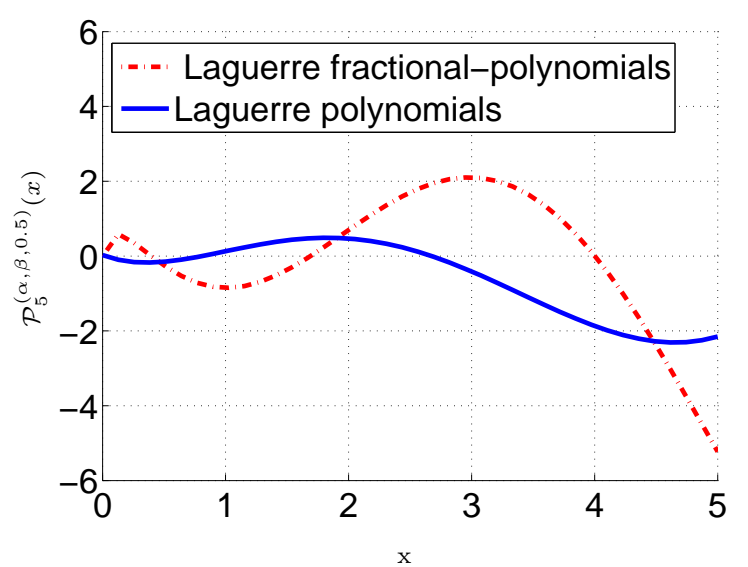

$\Lambda_{5}=2.1112, \mu=0.5$

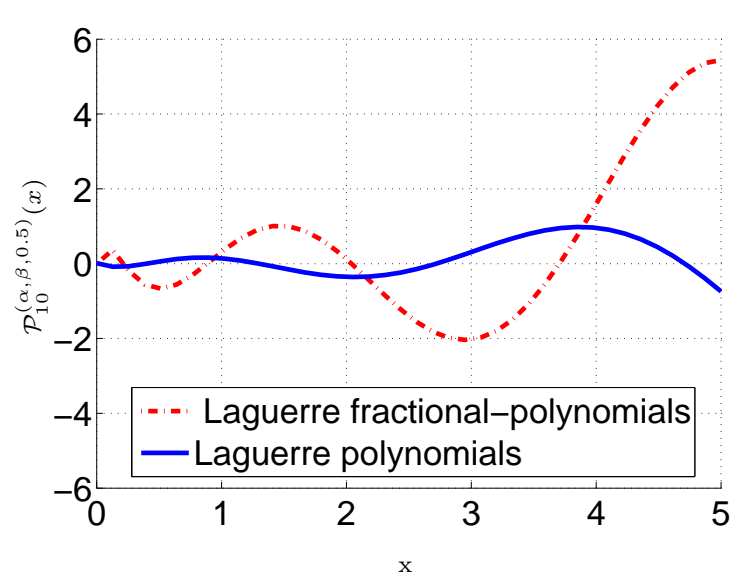

$\Lambda_{10}=3.0746, \mu=0.5$

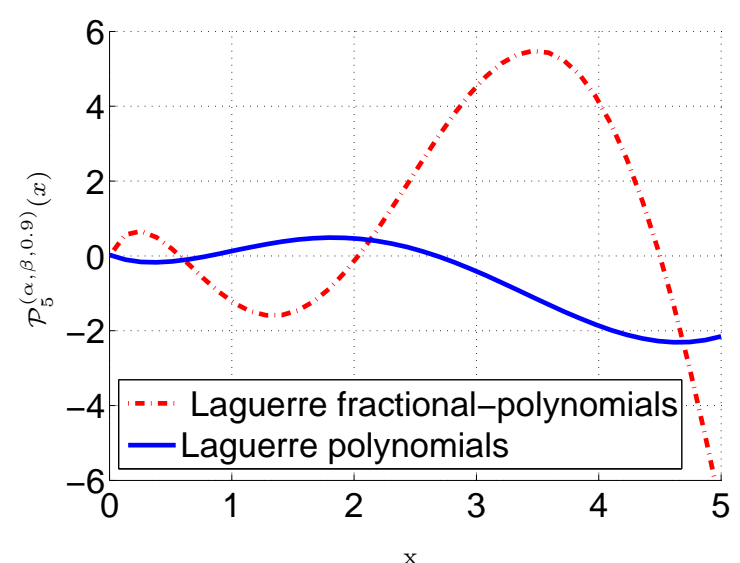

$\Lambda_{5}=4.8180, \mu=0.99$

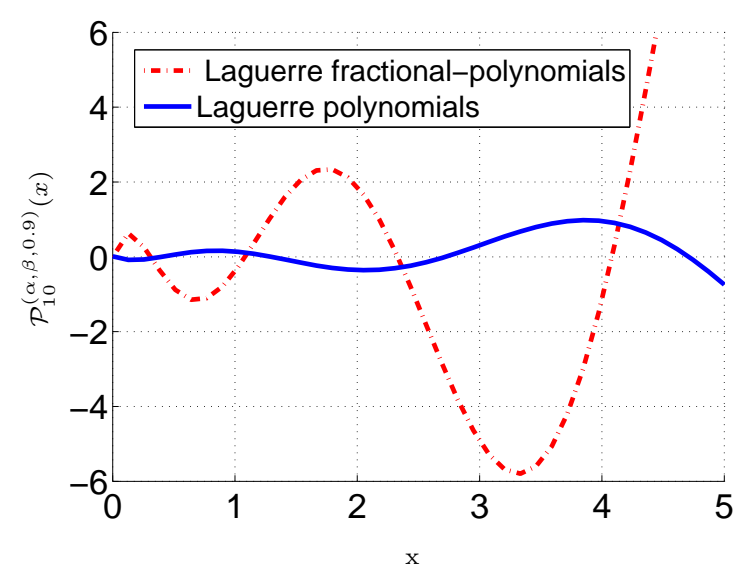

$\Lambda_{10}=9.6709, \mu=0.99$

Figure 2: Eigenfunctions of $\operatorname{SFSLP}, \mathcal{P}_{n}^{(\alpha, \beta, \mu)}(x)$, versus $x$, for $n=1$ (first row), $n=2$ (second row), $n=5$ (third row), and $n=10$ (last row), corresponding to the fractional-order $\mu=0.5$ (left column) and $\mu=0.99$ (right column). Here, we take the same values $\alpha=-0.9$ and $\beta=1$, as shown in Fig. 1 
or equivalently

$$
\begin{aligned}
& \hat{v}_{k}=\frac{-1}{\Lambda_{k} \Theta_{k}^{\alpha, \beta, \mu}} \int_{0}^{\infty} \mathcal{P}_{k}^{(\alpha, \beta, \mu)}(x) L^{\alpha, \beta ; \mu} v(x) d x, \\
& \left|\hat{v}_{k}\right| \leq \frac{1}{\Lambda_{k} \Theta_{k}^{\alpha, \beta, \mu}}\left[\int_{0}^{\infty}\left(\mathcal{P}_{k}^{(\alpha, \beta, \mu)}(x)\right)^{2} W^{(\alpha, \beta, \mu)}(x) d x\right]^{\frac{1}{2}}\left[\int_{0}^{\infty}\left(v_{1}(x)\right)^{2} W(x) d x\right]^{\frac{1}{2}}=\frac{\rho}{\left|\Lambda_{k}\right|}\left\|v_{1}(x)\right\|_{L_{W}^{2}},
\end{aligned}
$$

where $v_{1}(x) \equiv L^{\alpha, \beta ; \mu} v(x) / W^{(\alpha, \beta, \mu)}(x)$. By carrying out the fractional integration-by-parts another $(m-1)$ times, and setting $v_{m}(x) \equiv L^{\alpha, \beta ; \mu} v_{m-1}(x) / W^{(\alpha, \beta, \mu)}(x)$, we obtain

$$
\left|\hat{v}_{k}\right| \leq \frac{\rho}{\left|\Lambda_{k}\right|^{m}}\left\|v_{m}(x)\right\|_{L_{W}^{2}} .
$$

Consequently, if the function $v(x) \in C^{\infty}[0, \infty)$, we recover the exponential decay of the expansion coefficients $\hat{v}_{k}$.

\subsection{Optimal approximation for the generalized Laguerre fractional-polynomials}

We now turn to several orthogonal projections which are frequently using in the generalized Laguerre fractionalpolynomials spectral methods.

Consider the $L_{W^{(\alpha, \beta, \mu)}}^{2}\left(\mathbb{R}_{+}\right)$-orthogonal projection upon $F_{N}^{(\alpha, \beta, \mu)}\left(\mathbb{R}_{+}\right)$, defined by

$$
\left(I_{N}^{(\alpha, \beta, \mu)} v-v, u_{N}\right)_{W^{(\alpha, \beta, \mu)}}=0, \quad u_{N} \in F_{N}^{(\alpha, \beta, \mu)}\left(\mathbb{R}_{+}\right) .
$$

By definition, we have

$$
I_{N}^{(\alpha, \beta, \mu)} v(x)=\sum_{n=1}^{N} \hat{v}_{n}^{(\alpha, \beta, \mu)} \mathcal{P}_{n}^{(\alpha, \beta, \mu)}(x) .
$$

By the orthogonality 3.52 ,

$$
\int_{0}^{\infty}{ }^{C} \mathcal{D}^{\breve{\alpha}+k} \mathcal{P}_{n}^{(\alpha, \beta, \mu)}(x){ }^{C} \mathcal{D}^{\breve{\alpha}+k} \mathcal{P}_{m}^{(\alpha, \beta, \mu)}(x) w^{k, \beta} d x=\chi_{n, k}^{\alpha, \beta, \mu} \delta_{n, m},
$$

where

$$
\chi_{n, k}^{\alpha, \beta, \mu}=\frac{\beta^{2 k} \Gamma(n-\alpha+\mu-1)^{2}}{\Gamma(n)^{2}} \kappa_{n-k}^{k, \beta}=\frac{\beta^{k-1} \Gamma(n-\alpha+\mu-1)^{2}}{\Gamma(n)(n-k) !},
$$

and there holds the Parseval identity:

$$
\|v\|_{W^{(\alpha, \beta, \mu)}}^{2}=\sum_{n=1}^{\infty} \Theta_{n}^{\alpha, \beta, \mu}\left|\hat{v}_{n}^{(\alpha, \beta, \mu)}\right|^{2} .
$$

Remark 4.1 It is worthwhile to point out that for $\alpha>-1$ and $\breve{\alpha}=-\alpha+\mu-1$, we have

$$
\left({ }^{C} \mathcal{D}^{\breve{\alpha}+k}\left(I_{N}^{(\alpha, \beta, \mu)} v-v\right), D^{k} Q_{N}\right)_{w^{k, \beta}}=0, \quad \forall Q_{N} \in \mathbb{P}_{N} .
$$

Notice that

$$
\left(I_{N}^{(\alpha, \beta, \mu)} v-v\right)(x)=\sum_{n=N+1}^{\infty} \hat{v}_{n}^{(\alpha, \beta, \mu)} \mathcal{P}_{n}^{(\alpha, \beta, \mu)}(x),
$$


and $\mathbb{P}_{N}=\operatorname{span}\left\{L_{n}^{(0, \beta)}(x): 0 \leq n \leq N\right\}$ and ${ }^{C} \mathcal{D}^{\breve{\alpha}+k}={ }^{C} \mathcal{D}^{\breve{\alpha}} D^{k}$, we obtain (4.17) from (3.64), (3.68) and the orthogonality of the classical generalized Laguerre polynomials, we notice that $W^{\alpha, \beta, \mu}(x)=w^{\alpha-\mu+1, \beta}(x)$, $w^{\alpha, \beta}(x)$ is the weight function of the family of Laguerre polynomials.

To characterize the regularity of $v$, we introduce the non-uniformly weighted space involving fractional derivatives:

$$
\mathbf{A}_{\alpha, \beta, \mu}^{m}\left(\mathbb{R}_{+}\right):=\left\{v \in L_{W^{(\alpha, \beta, \mu)}}^{2}\left(\mathbb{R}_{+}\right):{ }^{C} \mathcal{D}^{\breve{\alpha}+k} v \in L_{w^{(k, \beta)}}^{2}\left(\mathbb{R}_{+}\right) \text {for } 0 \leq k \leq m\right\}, \quad m \in \mathbb{N}_{0} .
$$

By (4.13), 4.14 and 4.18), we have that

$$
{ }^{C} \mathcal{D}^{\breve{\alpha}+k}\left(I_{N}^{(\alpha, \beta, \mu)} v-v\right)(x)=\sum_{n=N+1}^{\infty} \frac{(-\beta)^{k} \Gamma(n-\alpha+\mu-1)}{\Gamma(n)} \hat{v}_{n}^{(\alpha, \beta, \mu)} L_{n-k-1}^{k, \beta}(x),
$$

whence

$$
\left\|{ }^{C} \mathcal{D}^{\breve{\alpha}+k}\left(I_{N}^{(\alpha, \beta, \mu)} v-v\right)(x)\right\|_{w^{(k, \beta)}}^{2}=\sum_{j=N+1}^{\infty} \chi_{j, k}^{\alpha, \beta, \mu}\left|\hat{v}_{j}^{(\alpha, \beta, \mu)}\right|^{2} .
$$

On the other hand,

$$
\left\|{ }^{C} \mathcal{D}^{\breve{\alpha}+k} v(x)\right\|_{w^{(k, \beta)}}^{2}=\sum_{j=k}^{\infty} \chi_{j, k}^{\alpha, \beta, \mu}\left|\hat{v}_{j}^{(\alpha, \beta, \mu)}\right|^{2} .
$$

We have the following basic result.

Theorem 4.1 let $v \in \mathbf{A}_{\alpha, \beta, \mu}^{m}\left(\mathbb{R}_{+}\right)$, with $m \in N_{0}$

- For $0 \leq k \leq m \leq N$,

$$
\left\|{ }^{C} \mathcal{D}^{\breve{\alpha}+k}\left(I_{N}^{(\alpha, \beta, \mu)} v-v\right)(x)\right\|_{w^{(k, \beta)}} \leq c \beta^{(k-m) / 2} \sqrt{\frac{(N-m+1) !}{(N-k+1) !}}\left\|^{C} \mathcal{D}^{\breve{\alpha}+m} v(x)\right\|_{w^{(m, \beta)}} .
$$

In particular, if $m$ is fixed, then

$$
\left\|{ }^{C} \mathcal{D}^{\breve{\alpha}+k}\left(I_{N}^{(\alpha, \beta, \mu)} v-v\right)(x)\right\|_{w^{(k, \beta)}} \leq c(\beta N)^{(k-m) / 2}\left\|{ }^{C} \mathcal{D}^{\breve{\alpha}+m} v(x)\right\|_{w^{(m, \beta)}} .
$$

- For $0 \leq m \leq N$, we also have the $L_{W^{(\alpha, \beta, \mu)}}^{2}$ estimates:

$$
\left\|\left(I_{N}^{(\alpha, \beta, \mu)} v-v\right)(x)\right\|_{W^{(\alpha, \beta, \mu)}} \leq c \beta^{(\alpha-\mu-m+1) / 2} \sqrt{\frac{(N-m+1) !}{(N-\alpha+\mu) !}}\left\|^{C} \mathcal{D}^{\breve{\alpha}+m} v(x)\right\|_{w^{(m, \beta)}} .
$$

In particular, if $m$ is fixed, then

$$
\left\|\left(I_{N}^{(\alpha, \beta, \mu)} v-v\right)(x)\right\|_{W^{(\alpha, \beta, \mu)}} \leq c(\beta N)^{(\alpha-\mu-m+1) / 2}\left\|{ }^{C} \mathcal{D}^{\breve{\alpha}+m} v(x)\right\|_{w^{(m, \beta)}} .
$$

Proof. By 4.13, 4.12) and 4.21,

$$
\left\|{ }^{C} \mathcal{D}^{\breve{\alpha}+k}\left(I_{N}^{(\alpha, \beta, \mu)} v-v\right)(x)\right\|_{w^{(k, \beta)}}^{2}=\sum_{j=N+1}^{\infty} \chi_{j, k}^{\alpha, \beta, \mu}\left|\hat{v}_{j}^{(\alpha, \beta, \mu)}\right|^{2}=\sum_{j=N+1}^{\infty} \frac{\chi_{j, k}^{\alpha, \beta, \mu}}{\chi_{j, m}^{\alpha, \beta, \mu}} \chi_{j, m}^{\alpha, \beta, \mu}\left|\hat{v}_{j}^{(\alpha, \beta, \mu)}\right|^{2} .
$$

We find that

$$
\frac{\chi_{N+1, k}^{\alpha, \beta, \mu}}{\chi_{N+1, m}^{\alpha, \beta, \mu}} \leq c \beta^{k-m} \frac{(N-m+1) !}{(N-k+1) !}
$$


Therefore, a combination of (4.27), (4.28) and 4.21) yields that

$$
\left\|{ }^{C} \mathcal{D}^{\breve{\alpha}+k}\left(I_{N}^{(\alpha, \beta, \mu)} v-v\right)(x)\right\|_{w^{(k, \beta)}} \leq c \beta^{(k-m) / 2} \sqrt{\frac{(N-m+1) !}{(N-k+1) !}}\left\|{ }^{C} \mathcal{D}^{\breve{\alpha}+m} v(x)\right\|_{w^{(m, \beta)}},
$$

which implies result 4.23). If $m$ is fixed, then we find that

$$
\frac{\chi_{N+1, k}^{\alpha, \beta, \mu}}{\chi_{N+1, m}^{\alpha, \beta}} \leq c \beta^{m-k} \frac{(N-m+1) !}{(N-k+1) !} \leq c(\beta)^{(m-k)}(N)^{(k-m)} .
$$

Hence

$$
\left\|{ }^{C} \mathcal{D}^{\breve{\alpha}+k}\left(I_{N}^{(\alpha, \beta, \mu)} v-v\right)(x)\right\|_{w^{(k, \beta)}} \leq c(\beta N)^{(k-m) / 2}\left\|{ }^{C} \mathcal{D}^{\breve{\alpha}+m} v(x)\right\|_{w^{(m, \beta)}},
$$

which implies result (4.24).

The $L_{W(\alpha, \beta, \mu)}^{2}$ estimates can be obtained by using the same argument. We sketch the derivation below. By 4.16 and 4.21,

$$
\begin{aligned}
\left\|\left(I_{N}^{(\alpha, \beta, \mu)} v-v\right)(x)\right\|_{W^{(\alpha, \beta, \mu)}}^{2} & =\sum_{j=N+1}^{\infty} \theta_{j}^{\alpha, \beta, \mu}\left|\hat{v}_{n}^{(\alpha, \beta, \mu)}\right|^{2}=\sum_{j=N+1}^{\infty} \frac{\theta_{j}^{\alpha, \beta, \mu}}{\chi_{j, m}^{\alpha, \beta, \mu}} \chi_{j, m}^{\alpha, \beta, \mu}\left|\hat{v}_{j}^{(\alpha, \beta, \mu)}\right|^{2} \\
& \leq \frac{\theta_{N+1}^{\alpha, \beta, \mu}}{\chi_{N+1, m}^{\alpha, \beta, \mu}} \sum_{j=N+1}^{\infty} \chi_{j, m}^{\alpha, \beta, \mu}\left|\hat{v}_{j}^{(\alpha, \beta, \mu)}\right|^{2} .
\end{aligned}
$$

By the Stirling formula, $\Gamma(k+1)=\sqrt{2 \pi k} k^{k} e^{-k}\left(1+\mathcal{O}\left(k^{\frac{-1}{5}}\right)\right)$, we find that

$$
\frac{\theta_{N+1}^{\alpha, \beta, \mu}}{\chi_{N+1, m}^{\alpha, \beta, \mu}}=\frac{\Gamma(N-\alpha+\mu)}{\beta^{-\alpha+\mu}(N) !} \frac{\Gamma(N+1)}{\Gamma(N-\alpha+\mu)^{2}} \frac{\beta^{1-m}(N-m+1) !}{\Gamma(N+1)} \leq c \beta^{(\alpha-\mu-m+1)} \frac{(N-m+1) !}{(N-\alpha+\mu) !} .
$$

Hence

$$
\left\|\left(I_{N}^{(\alpha, \beta, \mu)} v-v\right)(x)\right\|_{W^{(\alpha, \beta, \mu)}} \leq c \beta^{(\alpha-\mu-m+1) / 2} \sqrt{\frac{(N-m+1) !}{(N-\alpha+\mu) !}}\left\|^{C} \mathcal{D}_{x}^{\breve{\alpha}+m} v(x)\right\|_{w^{(m, \beta)}},
$$

which implies result (4.24). In particular, if $m$ is fixed, then

$$
\frac{\theta_{N+1}^{\alpha, \beta, \mu}}{\chi_{N+1, m}^{\alpha, \beta, \mu}} \leq c(\beta N)^{(\alpha-\mu-m+1) / 2}
$$

\section{$5 \quad$ PG spectral methods}

In this section, we examine three linear steady-state problems on a semi-infinite interval: (i) the space-fractional advection equation on half line, (ii) the space-fractional the space-fractional diffusion equation on half line and (iii) the space-fractional advection-diffusion equation on half line. Here, we take a step-by-step approach to show how the PG methods can be employed to solve different problems with almost the same ease.

We establish our variational form in the trial space $F_{N}^{(\alpha, \beta, \nu)}$ and test space $P_{N}^{\alpha, \beta}\left(\mathbb{R}_{+}\right)=e^{-\beta x} L_{n}^{-\alpha-1, \beta}(x)$ with weight $W^{(\alpha, \beta, \nu)}$ for fractional advection equation and $F_{N}^{(\alpha, \beta, \nu)}$ and $P_{N}^{\alpha+1, \beta}\left(\mathbb{R}_{+}\right)$with weight $W^{(\alpha+1, \beta, \nu)}$ for fractional diffusion equation and fractional advection-diffusion equation on half line. 


\subsection{Fractional initial value problems (FIVPs)}

As a first example, we consider the FIVP of order $\nu \in(0,1)$ on half line:

$$
{ }^{C} \mathcal{D}^{\nu} v(x)=f(x), \quad x \in \mathbb{R}_{+}, \quad v(0)=0,
$$

where $f \in L^{2}\left(\mathbb{R}_{+}\right)$.

The spectral PG scheme is to find $v_{N} \in F_{N}^{(\alpha, \beta, \mu)}\left(\mathbb{R}_{+}\right)$, such that

$$
\left({ }^{C} \mathcal{D}^{\nu} v_{N}, u_{N}\right)=\left(f(x), u_{N}\right), \quad \forall u_{N} \in \mathbb{P}_{N} .
$$

The following error estimate shows the spectral accuracy of this generalized Laguerre fractional-polynomials spectral PG approximation.

Theorem 5.1 Let $v$ and $v_{N}$ be the solution of 5.1 and (5.2), respectively. If $f^{(k)} \in L_{w^{(k, k)}}^{2}\left(\mathbb{R}_{+}\right)$for all $0 \leq k \leq m$, then we have that for $0 \leq m \leq N$,

$$
\left\|{ }^{C} \mathcal{D}^{\nu}\left(v-v_{N}\right)(x)\right\| \leq c(\beta N)^{(-m) / 2}\left\|f^{(m)}\right\|_{w^{(m, \beta)}},
$$

where $c$ is a positive constant independent of $v, N$ and $m$.

Proof. Let $I_{N}^{(\alpha, \beta, \mu)} v$ be the same as in 4.13) for $0<\nu<1$. By 4.17) (with $k=0$ )

$$
\left({ }^{C} \mathcal{D}^{\nu}\left(I_{N}^{(\alpha, \beta, \mu)} v-v_{N}\right), u\right)=0, \quad \forall u \in \mathbb{P}_{N} .
$$

Then by (5.1),

$$
\left(f-{ }^{C} \mathcal{D}^{\nu} I_{N}^{(\alpha, \beta, \mu)} v, u\right)=\left({ }^{C} \mathcal{D}^{\nu} v-{ }^{C} \mathcal{D}^{\nu} I_{N}^{(\alpha, \beta, \mu)} v, u\right) .
$$

Let $I_{N} f$ be the $L^{2}$-orthogonal projection of $f$ upon $\mathbb{P}_{N}$. We infer from 5.5 that

$$
{ }^{C} \mathcal{D}^{\nu} I_{N}^{(\alpha, \beta, \mu)} v(x)=I_{N} f .
$$

On the other hand, by $(5.2)$

$$
{ }^{C} \mathcal{D}^{\nu} v_{N}(x)=I_{N} f .
$$

Thus, we have

$$
{ }^{C} \mathcal{D}^{\nu}\left(I_{N}^{(\alpha, \beta, \mu)} v(x)-v_{N}\right)=0 .
$$

Therefore, it follows from Theorem 4.1 (with $k=0, \breve{\alpha}=\nu$ )that

$$
\left\|{ }^{C} \mathcal{D}^{\nu}\left(v-v_{N}\right)(x)\right\|=\left\|{ }^{C} \mathcal{D}^{\nu}\left(v-I_{N}^{(\alpha, \beta, \mu)} v\right)(x)\right\| \leq c(\beta N)^{(-m) / 2}\left\|{ }^{C} \mathcal{D}^{\nu+m} v\right\|_{w^{(m, \beta)}} \leq c(\beta N)^{(-m) / 2}\left\|f^{(m)}\right\|_{w^{(m, \beta)}}
$$

\subsubsection{Steady-state fractional advection problem}

We consider the factional initial value problem of order $\nu \in(0,1)$ on half line:

$$
{ }^{C} \mathcal{D}^{\nu} v(x)=f(x), \quad x \in \mathbb{R}_{+}, \quad v(0)=0,
$$

where $f \in L^{2}\left(\mathbb{R}_{+}\right)$.

The spectral PG scheme is to find $v_{N} \in F_{N}^{(\alpha, \beta, \nu)}\left(\mathbb{R}_{+}\right)$, such that

$$
\left({ }^{C} \mathcal{D}^{\nu} v_{N}, u_{N}\right)=\left(f(x), u_{N}\right), \quad \forall u_{N} \in \mathcal{P}_{N}^{\alpha, \beta}\left(\mathbb{R}_{+}\right) .
$$


By means of property 2.11, we obtain

$$
\left(v_{N}(x),{ }^{W} \mathcal{D}^{\nu} u_{N}\right)=\left(f(x), u_{N}\right) .
$$

Using generalized Laguerre fractional basis, we can write

$$
v_{N}(x)=\sum_{i=1}^{N} \hat{v}_{i}^{(\alpha, \beta, \mu)} \mathcal{P}_{i}^{(\alpha, \beta, \mu)}(x),
$$

substitute (5.13) into $(5.12)$ and by carrying out the Weyl fractional derivative in the bracket using $(3.28)$, we obtain

$$
\beta^{\nu} \sum_{i=1}^{N} \hat{v}_{i}^{(\alpha, \beta, \mu)} \int_{0}^{\infty} e^{(-\beta x)} x^{-\alpha+\nu-1} L_{i-1}^{-\alpha+\nu-1, \beta}(x) L_{k}^{-\alpha+\nu-1, \beta}(x) d x=\int_{0}^{\infty} e^{(-\beta x)} L_{k}^{-\alpha-1, \beta}(x) f(x) d x,
$$

which yields stiffness matrix on the left-hand side, whose diagonal entries are given by $\kappa_{(k-1)}^{-\alpha+\nu-1, \beta}$. Consequently, we obtain the expansion coefficients as

$$
\hat{v}_{i}^{(\alpha, \beta, \mu)}=\frac{1}{\kappa_{(k-1)}^{-\alpha+\nu-1, \beta}} \int_{0}^{\infty} e^{(-\beta x)} L_{k}^{-\alpha-1, \beta}(x) f(x) d x .
$$

In Fig. 3, we present numerical results obtained using the PG spectral method to steady-state fractional advection problem, corresponding to $\nu=1 / 10$ and 9/10. Here we consider two different exact solutions:

(a). Exponential decay without oscillations at infinity.

$$
f(x)=x^{\frac{19}{20}} e^{-x} .
$$

(b). Algebraic decay without oscillations at infinity.

$$
f(x)=\frac{x^{\frac{19}{20}}}{(1+x)^{h}}, \quad h>1 .
$$

In many applications with the above formulation is that the decay algebraically or exponentially weight associated with the variational formulation renders the approximate solution useless for large $x$. Thus the Laguerre fractional polynomials are not suitable for practical numerical computations. on which the Laguerre fractional approximations are often based, is not well posed. One possible way to remedy this deficiency is to find a suitable variable transform such that the weighted variational formulation of the transformed equation becomes well posed.

Multiplying (5.1] by $u_{N} \in P_{N}^{\alpha, \beta}$ and integrating Integrating over the interval $[0, \infty)$, we obtain

$$
\left({ }^{C} \mathcal{D}^{\nu} v_{N}, u_{N}\right)=\left(f(x), u_{N}\right), \quad \forall u_{N} \in P_{N}^{\alpha, \beta}\left(\mathbb{R}_{+}\right) .
$$

We make the variable transform, setting

$$
v(x)=e^{(-\beta x)} \bar{v}(x) .
$$

By means of property 2.11, we obtain

$$
\left(e^{(-\beta x)} \bar{v}_{N}(x),{ }^{W} \mathcal{D}^{\nu} u_{N}\right)=\left(f(x), u_{N}\right), \quad \forall \bar{v}_{N} \in F_{N}^{(\alpha, \beta, \nu)}\left(\mathbb{R}_{+}\right),
$$

substitute (5.13) into (5.18) and by carrying out the Weyl fractional derivative in the bracket using (3.28), we obtain

$$
\beta^{\nu} \sum_{i=1}^{N} \hat{v}_{i}^{(\alpha, \beta, \mu)} \int_{0}^{\infty} e^{(-2 \beta x)} x^{-\alpha+\nu-1} L_{i-1}^{-\alpha+\nu-1, \beta}(x) L_{k}^{-\alpha+\nu-1, \beta}(x) d x=\int_{0}^{\infty} e^{(-\beta x)} L_{k}^{-\alpha-1, \beta}(x) f(x) d x,
$$



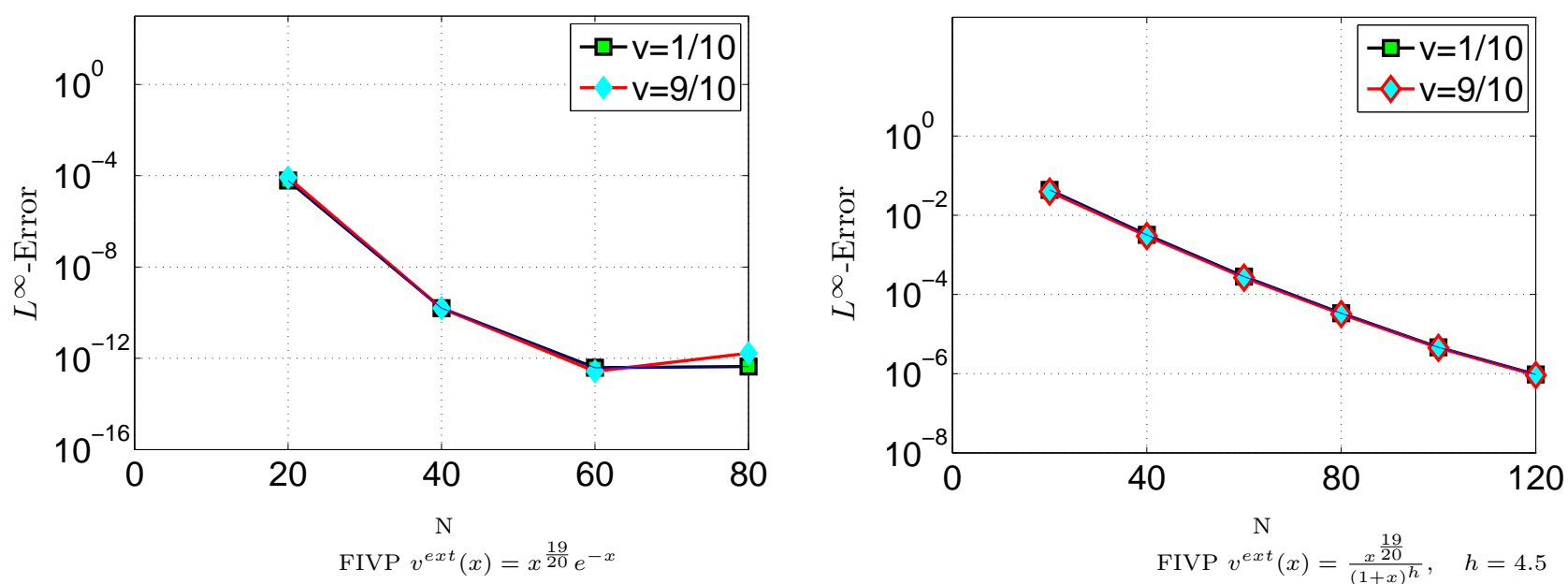

Figure 3: Steady-state fractional advection problem: log-linear $L^{\infty}$-norm error of the numerical solution to ${ }^{C} \mathcal{D}^{\nu} v(x)=$ $f(x), x \in \mathbb{R}_{+}$, versus $N$ corresponds to the fractional order $\nu=1 / 10$ and $9 / 10$.

which yields stiffness matrix on the left-hand side, whose entries are given by

$$
S_{i, k}=\frac{\beta^{\nu} \Gamma(i+k-\alpha+\nu-1)}{(k) !(i-1) !(2 \beta)^{(i+k-\alpha+\nu-1)}} .
$$

Consequently, we obtain the expansion coefficients as

$$
\hat{v}_{i}^{(\alpha, \beta, \mu)}=\frac{1}{S_{i, k}} \int_{0}^{\infty} e^{(-\beta x)} L_{k}^{-\alpha-1, \beta}(x) f(x) d x .
$$

In Fig. 4. we present numerical results obtained using the PG spectral method to steady-state fractional advection problem for large $x$, corresponding to $\nu=1 / 10$ and $9 / 10$. Having the same analytical solution taken in the previous case.

\subsection{Fractional boundary value problems(FBVPs)}

We consider the FBVP of order $\nu \in(0,1)$

$$
{ }^{C} \mathcal{D}^{1+\nu} v(x)=f(x), \quad x \in \mathbb{R}_{+}, \quad v(0)=0, \quad \lim _{x \rightarrow \infty} v(x)=0,
$$

where $f \in L^{2}\left(\mathbb{R}_{+}\right)$. Multiplying $\left(5.22\right.$ by $u_{N} \in P_{N}^{\alpha+1, \beta}\left(\mathbb{R}_{+}\right)$and integrating over the interval $[0, \infty)$, we obtain

$$
\left({ }^{C} \mathcal{D}^{1+\nu} v_{N}, u_{N}\right)=\left(f(x), u_{N}\right), \quad \forall v_{N} \in F_{N}^{(\alpha, \beta, \nu)}\left(\mathbb{R}_{+}\right) .
$$

We make the variable transform, setting

$$
v(x)=e^{(-\beta x)} \bar{v}(x) .
$$

By means of property 2.11, we obtain

$$
\left(e^{(-\beta x)} \bar{v}_{N}(x),{ }^{W} \mathcal{D}^{1+\nu} u_{N}\right)=\left(f(x), u_{N}\right), \quad \forall \bar{v}_{N} \in F_{N}^{(\alpha, \beta, \nu)}\left(\mathbb{R}_{+}\right)
$$

substitute 5.13 into 5.25 and by carrying out the Weyl fractional derivative in the bracket using (3.28), we obtain

$$
\beta^{\nu+1} \sum_{i=1}^{N} \hat{v}_{i}^{(\alpha, \beta, \mu)} \int_{0}^{\infty} e^{(-2 \beta x)} x^{-\alpha+\nu-1} L_{i-1}^{-\alpha+\nu-1, \beta}(x) L_{k}^{-\alpha+\nu-1, \beta}(x) d x=\int_{0}^{\infty} e^{(-\beta x)} L_{k}^{-\alpha-2, \beta}(x) f(x) d x,
$$



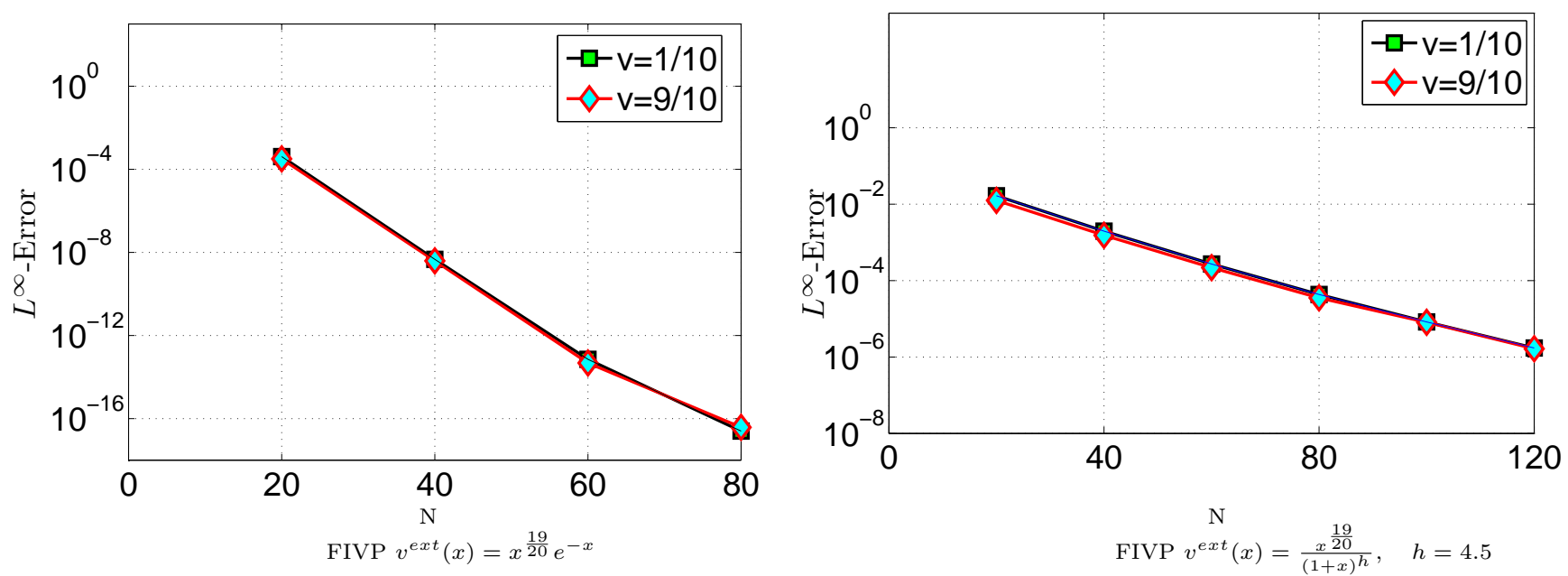

Figure 4: Steady-state fractional advection problem: log-linear $L^{\infty}$-norm error of the numerical solution to ${ }^{C} \mathcal{D}^{\nu} v(x)=$ $f(x), x \in \mathbb{R}_{+}$, versus $N$ corresponds to the fractional order $\nu=1 / 10$ and $9 / 10$.

which yields stiffness matrix on the left-hand side, whose entries are given by

$$
S_{i, k}=\frac{\beta^{\nu+1} \Gamma(i+k-\alpha+\nu-1)}{(k) !(i-1) !(2 \beta)^{(i+k-\alpha+\nu-1)}} .
$$

Consequently, we obtain the expansion coefficients as

$$
\hat{v}_{i}^{(\alpha, \beta, \mu)}=\frac{1}{S_{i, k}} \int_{0}^{\infty} e^{(-\beta x)} L_{k}^{-\alpha-2, \beta}(x) f(x) d x .
$$

\subsubsection{Steady-state fractional diffusion problem}

We examine a higher order FODE that is a space-fractional diffusion equation of order $1+\nu, \nu \in(0,1)$ on half line,

$$
{ }^{C} \mathcal{D}^{1+\nu} v(x)=f(x), \quad x \in \mathbb{R}_{+}, \quad v(0)=0, \quad \lim _{x \rightarrow \infty} v(x)=0 .
$$

In Fig. 5. we present numerical results obtained using the PG spectral method to steady-state fractional diffusion problem on half line, corresponding to $\nu=1 / 10$ and 9/10. Having the same analytical solution taken in the previous case. In all aforementioned cases exponential convergence is observed

\subsubsection{Steady-state fractional advection-diffusion problem}

Here, we consider a two term equation that describes the dynamics of the steady-state fractional advectiondiffusion problem on half line. Particularly, we are interested in the following case:

$$
c_{1}{ }^{C} \mathcal{D}^{\nu} v(x)+c_{2}{ }^{C} \mathcal{D}^{1+\nu} v(x)=f(x), \quad x \in \mathbb{R}_{+}, \quad v(0)=0, \quad \lim _{x \rightarrow \infty} v(x)=0, \quad \nu \in(0,1) .
$$

Multiplying (5.30) by $u_{N} \in P_{N}^{\alpha+1, \beta}\left(\mathbb{R}_{+}\right)$and integrating over the interval $[0, \infty)$, we obtain

$$
\left(\left(c_{1}{ }^{C} \mathcal{D}^{\nu}+c_{2}{ }^{C} \mathcal{D}^{1+\nu}\right) v_{N}, u_{N}\right)=\left(f(x), u_{N}\right), \quad \forall v_{N} \in F_{N}^{(\alpha, \beta, \nu)}\left(\mathbb{R}_{+}\right) .
$$

We make the variable transform, setting

$$
v(x)=e^{(-\beta x)} \bar{v}(x),
$$



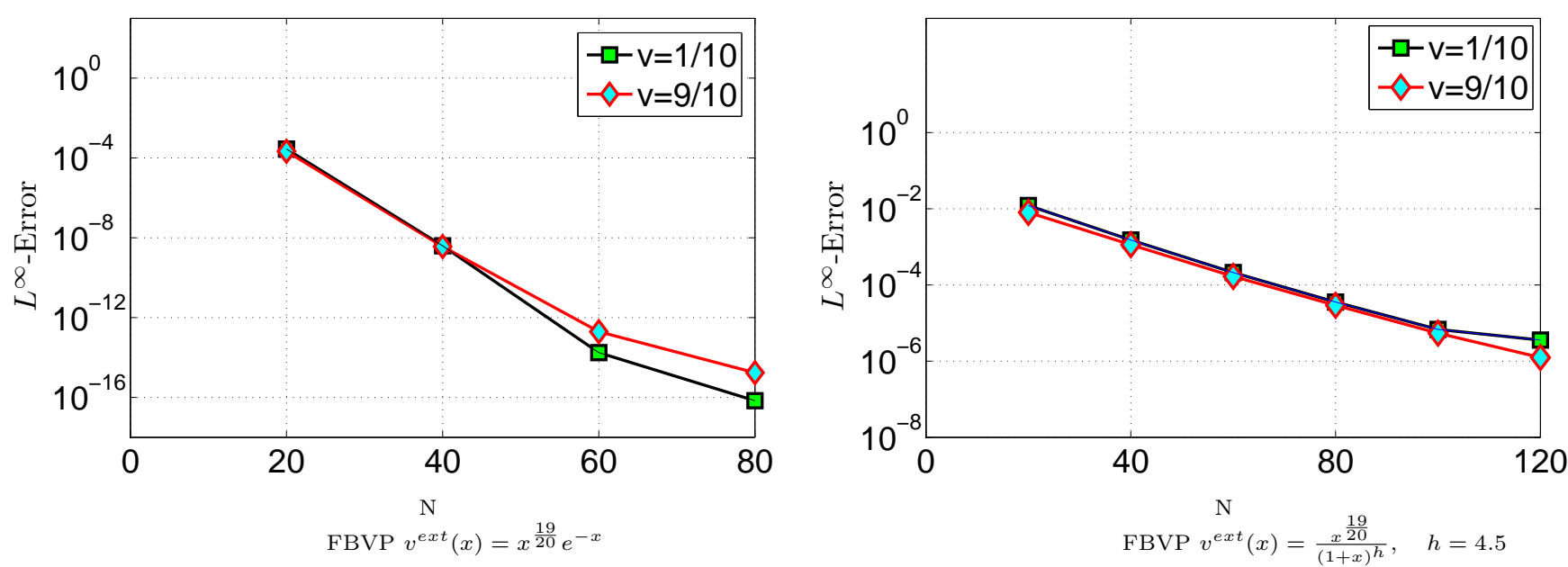

Figure 5: Steady-state fractional diffusion problem: log-linear $L^{\infty}$-norm error of the numerical solution to ${ }^{C} \mathcal{D}^{1+\nu} v(x)=$ $f(x), x \in \mathbb{R}_{+}$, versus $N$ corresponds to the fractional order $\nu=1 / 10$ and $9 / 10$.

the equation (5.31) becomes

$$
\begin{aligned}
& \left(\left(c_{1}{ }^{C} \mathcal{D}^{\nu}+c_{2}{ }^{C} \mathcal{D}^{1+\nu}\right) e^{(-\beta x)} \bar{v}_{N}(x), u_{N}\right)=\left(f(x), u_{N}\right), \\
& \left(c_{1}{ }^{C} \mathcal{D}^{\nu} e^{(-\beta x)} \bar{v}_{N}(x), u_{N}\right)+c_{2}\left({ }^{C} \mathcal{D}^{1+\nu} e^{(-\beta x)} \bar{v}_{N}(x), u_{N}\right)=\left(f(x), u_{N}\right) .
\end{aligned}
$$

By means of property (2.11), we obtain

$$
\left(c_{1} e^{(-\beta x)} \bar{v}_{N}(x),{ }^{W} \mathcal{D}^{\nu} u_{N}\right)+c_{2}\left(e^{(-\beta x)} \bar{v}_{N}(x),{ }^{W} \mathcal{D}^{1+\nu} u_{N}\right)=\left(f(x), u_{N}\right),
$$

substitute (5.13) into (5.35) and by carrying out the Weyl fractional derivative in the bracket using (3.28), we obtain

$$
\begin{aligned}
& c_{1} \beta^{\nu} \sum_{i=1}^{N} \hat{v}_{i}^{(\alpha, \beta, \mu)} \int_{0}^{\infty} e^{(-2 \beta x)} L_{i-1}^{-\alpha+\nu-1, \beta}(x) L_{k}^{-\alpha+\nu-2, \beta}(x) d x \\
& +c_{2} \beta^{\nu+1} \sum_{i=1}^{N} \hat{v}_{i}^{(\alpha, \beta, \mu)} \int_{0}^{\infty} e^{(-2 \beta x)} L_{i-1}^{-\alpha+\nu-1, \beta}(x) L_{k}^{-\alpha+\nu-1, \beta}(x) d x=\int_{0}^{\infty} e^{(-\beta x)} L_{k}^{-\alpha-2, \beta}(x) f(x) d x,
\end{aligned}
$$

which yields stiffness matrix on the left-hand side, whose entries are given by

$$
\mathcal{S}_{i, k}=\frac{1}{c_{1} \beta^{\nu} \sigma_{i, k}+c_{2} \beta^{\nu+1} \zeta_{i, k}},
$$

where

$$
\begin{aligned}
\sigma_{i, k} & =\frac{2 \Gamma(i+k-\alpha+\nu-1)}{(k) !(i-1) !(2 \beta)^{(i+k-\alpha+\nu-1)}}, \\
\zeta_{i, k} & =-\frac{\Gamma(i+k-\alpha+\nu-2)}{(k-1) !(i-1) !(2 \beta)^{(i+k-\alpha+\nu-2)}} .
\end{aligned}
$$



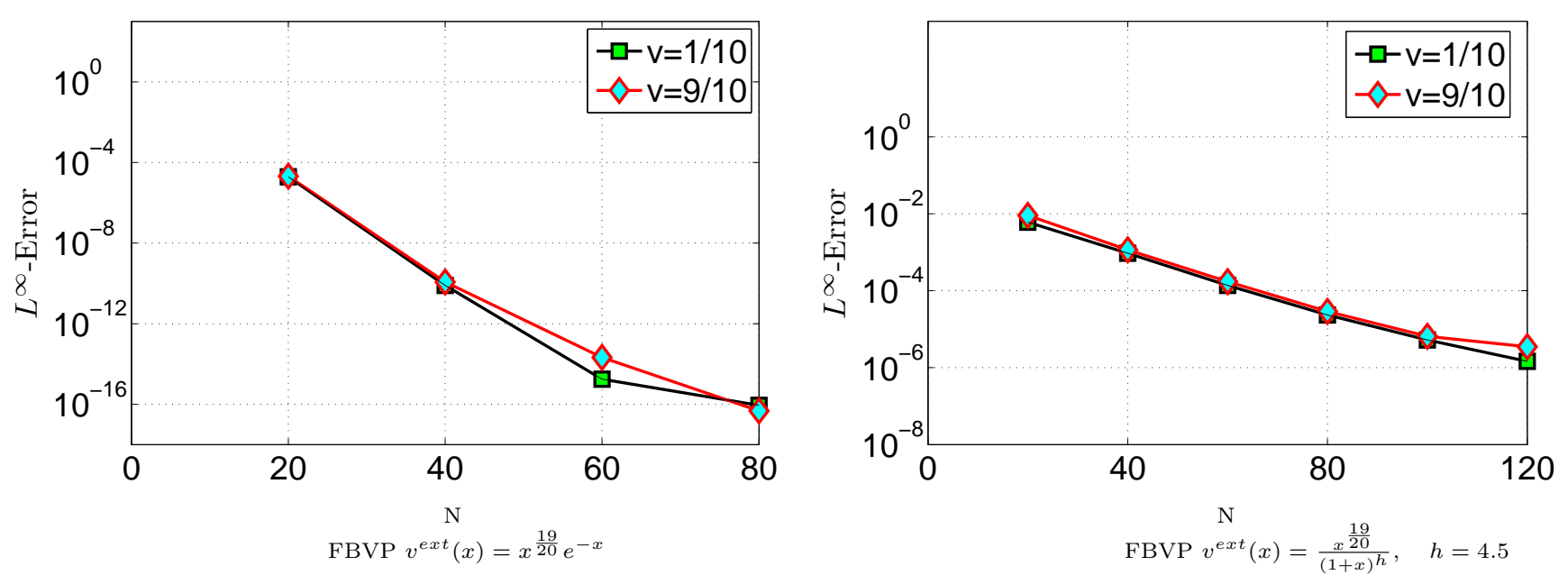

Figure 6: Steady-state fractional advection-diffusion problem: log-linear $L^{\infty}$-norm error of the numerical solution to ${ }^{C} \mathcal{D}^{\nu} v(x)+{ }^{C} \mathcal{D}^{1+\nu} v(x)=f(x), x \in \mathbb{R}_{+}$, versus $N$ corresponds to the fractional order $\nu=1 / 10$ and $9 / 10$.

Consequently, we obtain the expansion coefficients as

$$
\hat{v}_{i}^{(\alpha, \beta, \mu)}=\frac{1}{\mathcal{S}_{i, k}} \int_{0}^{\infty} e^{(-\beta x)} L_{k}^{-\alpha-2, \beta}(x) f(x) d x .
$$

In Fig. 6, we present the log-linear $L^{\infty}$-norm error of the numerical solution to $c_{1}{ }^{C} \mathcal{D}^{\nu} v(x)+c_{2}{ }^{C} \mathcal{D}^{1+\nu} v(x)=$ $f(x), x \in \mathbb{R}_{+}$, versus $N$, corresponding to $\nu=1 / 10$ and $9 / 10$. Having the same analytical solution taken in the previous case.

\section{Summary}

In this article, we have considered SFSLP of order $2 \mu$ with fractional differential operator associated to Weyl and Caputo type of the same fractional-order $\mu$. This choice is motivated by a proper fractional integration-by-parts. Also, we have obtained the analytical eigensolutions, and their recursive relation, to SFSLP which is defined as generalized Laguerre fractional-polynomials. These eigenfunctions were shown to be orthogonal with respect to the weight function, which were also hierarchical. The analysis of approximations by generalized Laguerre fractional-polynomials was derived. Such approximation plays an important role in the analysis of fractional spectral methods for FDEs. We have constructed a highly-accurate fractional spectral PG methods for a class of prototypical FDEs on half line, including FIVPs and FBVPs which have not been numerically studied before. We considered steady-state problems such as the space-fractional advection-diffusion problem on half line. Our numerical results confirmed the exponential convergence of PG methods.

\section{References}

[1] K. Diethelm, N. J. Ford, Analysis of fractional differential equations, J. Math. Anal. Appl. 265 (2002), pp. 229-248.

[2] A. A. Kilbass, H. M. Srivastava, J. J. Trujillo, Theory and Applications of Fractional Differential Equations, Elsevier, Amsterdam, 2006.

[3] M. Klimek, O. P. Agrawal, On a regular fractional Sturm-Liouville problem with derivatives of order in $(0,1)$, in: Proceedings of the 13th International Carpathian Control Conference (ICCC), July 2012, 978-1-45771868. 
[4] E. Barkai, R. Metzler, J. Klafter, From continuous time random walks to the fractional Fokker-Planck equation, Phys. Rev. E 61 (2000), pp. 132-138.

[5] N. Sugimoto, Burgers equation with a fractional derivative; hereditary effects on nonlinear acoustic waves, J. Fluid Mech. 225 (1991), pp. 631-653.

[6] B. I. Henry, S. L. Wearne, Fractional reaction-diffusion, Physica A 276 (2000), pp. 448-455.

[7] A. A. Kilbas, H. M. Srivastava, J. J. Trujillo, Theory and Applications of Fractional Differential Equations, Elsevier B. V., Amsterdam, 2006.

[8] I. Podlubny, Fractional Differential Equations, in: Mathematics in Science and Engineering, Academic Press, New York, London, Toronto, 198 (1999).

[9] S. G. Samko, A. A. Kilbas, O. I. Marichev, Fractional Integrals and Derivatives: Theory and Applications, Gordon and Breach, Yverdon, 1993.

[10] R. L. Magin, Fractional Calculus in Bioengineering, Begell House Inc., Redding, CT, 2006.

[11] X. Li, C. Xu, A space-time spectral method for the time fractional diffusion equation, SIAM J. Numer. Anal. 47 (2009), pp. 2108-2131.

[12] X. Li, C. Xu, Existence and uniqueness of the weak solution of the space-time fractional diffusion equation and a spectral method approximation, Commun. Comput. Phys. 8 (2010), pp. 1016-1051.

[13] M. M. Meerschaert, C. Tadjeran, Finite difference approximations for fractional advection-dispersion flow equations, J. Comput. Appl. Math. 172 (2004), pp. 65-77.

[14] S. B. Yuste, L. Acedo, An explicit finite difference method and a new von-Neumann-type stability analysis for fractional diffusion equations, SIAM J. Numer. Anal. 42 (2005), pp. 1862-1874.

[15] K. X. Wang, H. Wang, A fast characteristic finite difference method for fractional advection-diffusion equations, Adv. Water Resour. 34 (2011), pp. 810-816.

[16] G. Gao, Z. Sun, Y. Zhang, A finite difference scheme for fractional sub-diffusion equations on an unbounded domain using artificial boundary conditions, J. Comput. Phys. 231 (2012), pp. 2865-2879.

[17] W. Deng, Finite element method for the space and time fractional Fokker-Planck equation, SIAM J. Numer. Anal. 47 (2008), pp. 204-226.

[18] R. Gorenflo, F. Mainardi, D. Moretti, P. Paradisi, Time fractional diffusion: A discrete random walk approach, Nonlinear Dyn. 29 (2002), pp. 129-143.

[19] Z. Odibat, Rectangular decomposition method for fractional diffusion-wave equations, Appl. Math. Comput. 179 (2006), pp. 92-97.

[20] A. El-Sayed, S. Behiry, W. Raslan, Adomian's decomposition method for solving an intermediate fractional advection-dispersion equation, Comput. Math. Appl. 59 (2010), pp. 1759-1765.

[21] S. Momani, Z. Odibat, Homotopy perturbation method for nonlinear partial differential equations of fractional order, Phys. Lett. A 365 (2007), pp. 345-350.

[22] A. Yildirim, Analytical approach to Fokker-Planck equation with space- and time-fractional derivatives by means of the homotopy perturbation method, J. King Saud Univ. (Science) 22 (2010), pp. 257-264.

[23] P. Kumar, O. P. Agrawal, An approximate method for numerical solution of fractional differential equations, Signal Process 86 (2006), pp. 2602-261. 
[24] V. S. Ertürk, Computing eigenelements of Sturm-Liouville problems of fractional order via fractional differential transform method, Math. Compt. Appl. 16 (2011), pp. 712-720.

[25] Q. M. Al-Mdallal, An efficient method for solving fractional Sturm-Liouville problems, Chaos, Solitons , Fractals 40 (2009), pp. 183-189.

[26] A. Neamaty, R. Darzi, S. Zaree, B. Mohammadzadeh, Haar wavelet operational matrix of fractional order integration and its application for eigenvalues of fractional Sturm-Liouville problem, World Applied Sciences Journal 16 (2012), pp. 1668-1672.

[27] M. Zayernouri, G. E. Karniadakis, Exponentially accurate spectral and spectral element methods for fractional ODEs, J. Comput. Phys. 257 (2014), pp. 460-480.

[28] M. Zayernouri, G. E. Karniadakis, Fractional Sturm-Liouville eigen-problems: Theory and numerical approximations, J. Comput. Phys. 47 (2013), pp. 2108-2131.

[29] M. Zayernouri, G. E. Karniadakis, Fractional spectral collocation methods for linear and nonlinear variable order FPDEs, J. Comput. Phys. 293 (2014), pp. 312-338.

[30] A. Bueno-Orovio, D. Kay, K. Burrage, Fourier spectral methods for fractional-in-space reaction-diffusion equations. BIT Numer. Math. (2014), doi:10.1007/s10543-014-0484-2.

[31] E. H. Doha, A. H. Bhrawy, D. Baleanu, S. S. Ezz-Eldien, On shifted Jacobi spectral approximations for solving fractional differential equations, Appl. Math. Comput. 219 (2013), pp. 8042-8056.

[32] A. H. Bhrawy, M. A. Zaky, D. Baleanu, New numerical approximations for space-time fractional Burgers equations via a Legendre spectral-collocation method, Rom. Rep. Phys. 67 (2015), pp. 1-11.

[33] A. H. Bhrawy, E. H. Doha, S. S. Ezz-Eldien, M. A. Abdelkawy, A numerical technique based on the shifted Legendre polynomials for solving the time-fractional coupled KdV equation, Calcolo (2015), DOI 10.1007/s10092-014-0132-x.

[34] A. H. Bhrawy, and M. A. Abdelkawy, A Fully Spectral Collocation Approximation for Multi-Dimensional Fractional Schrödinger Equations, J. Comput. Phys. 294 (2015), pp. 462-483.

[35] A. H. Bhrawy, and M.A. Abdelkawy, A method based on the Jacobi tau approximation for solving multi-term time-space fractional partial differential equations, J. Comput. Phys. 281 (2015), pp. 876-895.

[36] M. Benchohra, N. Hamidi, Fractional order differential inclusions on the half-line, Surv. Math. Appl. 5 (2010), pp. 99-111.

[37] R. P. Agarwal, M. Benchohra, S. Hamani, S. Pinelas, Boundary value problems for differential equations involving Riemann-Liouville fractional derivative on the half-line, Dyn. Contin. Discrete Impuls. Syst. Ser. A Math. Anal. 18 (2011), pp. 235-244.

[38] A. Arara, M. Benchohra, N. Hamidi, J. J. Nieto, Fractional order differential equations on an unbounded domain, Nonlinear Anal. 72 (2010), pp. 580-586.

[39] T. Langlands, B. Henry, S. Wearne, Fractional cable equation models for anomalous electrodiffusion in nerve cells: infinite domain solutions, J. Math. Biol. 59 (2009), pp. 761-808.

[40] F. Huang, Analytical solution for the time-fractional telegraph equation, J. Appl. Math. (2009), 890158.

[41] D. Baleanu, A. H. Bhrawy, T. M. Taha, Two efficient generalized Laguerre spectral algorithms for fractional initial value problems, Abstr. Appl. Anal.(2013), doi:10.1155/2013/546502.

[42] D. Baleanu, A. H. Bhrawy, T. M. Taha, A modified generalized Laguerre spectral methods for fractional differential equations on the half line, Abstr. Appl. Anal. 2013 (2013), doi:10.1155/2013/413529. 
[43] A. H. Bhrawy, A. A. AL-Zahrani, Y. A. Alhamed, D. Baleanu, A New Generalized Laguerre-Gauss Collocation Scheme for Numerical Solution of Generalized Fractional Pantograph Equations, Romanian Reports Of Physics, 59 (7-8) (2014), pp. 646-657

[44] A. H. Bhrawy, T. M. Taha, J. A. T. Machado, A review of operational matrices and spectral techniques for fractional calculus, Nonlinear Dyn. (2015), doi:10.1007/ s11071-015-2087-0.

[45] C. Bernardi, Y. Maday, Spectral Methods, in Handbook of Numerical Analysis, Techniques of Scientific Computing, P. G. Ciarlet and J. L. Lions, eds., North-Holland, Amsterdam, 5 (1997), pp. 209-485.

[46] J. P. Boyd, Chebyshev and Fourier Spectral Methods, 2nd edn. Dover, New York, 2001.

[47] C. Canuto, M. Y. Hussaini, A. Quarteroni, A. Zang, Spectral Methods, Fundamentals in Single Domains, Springer, Berlin 2006.

[48] D. Funaro, Polynomial Approximation of Differential Equations, Springer-Verlag, Berlin 1992.

[49] B. -y. Guo, Spectral Methods and Their Applications, World Scientific, Singapore 1998.

[50] J. Shen, Stable and efficient spectral methods in unbounded domains using Laguerre functions, SIAM J. Numer. Anal. 38 (2000), pp. 1113-1133.

[51] B. -Y. Guo, J. Shen, Laguerre-Galerkin method for nonlinear partial differential equations on a semi-infinite interval, Numer. Math. 86 (2000), pp. 635-654.

[52] B. -Y. Guo, Tian-jun Wang, Composite generalized Laguerre-Legendre spectral method with domain decomposition and its application to Fokker-Planck equation in an finite channel, Math. Comp. 78 (2009), pp. 129-151.

[53] B. -Y. Guo, C. -L. Xu, Mixed Laguerre-Legendre pseudospectral method for incompressible fluid flow in an infinite strip, Math. Comp. 72 (2003), pp. 95-125.

[54] G. Mastroianni, G. Monegato, Nystrom interpolants based on zeros of Laguerre polynomials for some WeinerHopf equations, IMA J. Numer. Anal. 17 (1997), pp. 621-642.

[55] K. S. Miller, B. Ross, An Introduction to the Fractional Calculus and Fractional Differential Equations, Wiley, New York, 1993.

[56] K. S. Miller, The Weyl fractional calculus, Fractional Calculus and its Applications, Lecture Notes in Math., Springer-Verlag, New York, 457 (1975), pp. 80-89.

[57] R. Askey, J. Fitch, Integral representations for Jacobi polynomials and some applications, J. Math. Anal. Appl. 26 (1969), pp. 411-437.

[58] B. -Y. Guo, X. -Y. Zhang, A new generalized Laguerre spectral approximation and its applications, J. Comp. Appl. Math. 181 (2005), pp. 342-363. 